\title{
Variability of updated finite element models and their predictions consistent with vibration measurements
} \author{
Costas Papadimitriou ${ }^{1, *, \dagger}$, Evangelos Ntotsios ${ }^{1}$, Dimitrios Giagopoulos $^{2}$
and Sotirios Natsiavas \\ ${ }^{1}$ Department of Mechanical Engineering, University of Thessaly, Volos 38334, Greece \\ ${ }^{2}$ Department of Mechanical Engineering, Aristotle University, Thessaloniki 54124, Greece
}

\begin{abstract}
A case study on a small-scale laboratory vehicle frame is used to investigate the variability of the updated finite element (FE) models that arises from model and measurement errors and demonstrate the effect of this variability on response predictions. Conventional weighted modal residuals and recently introduced multi-objective identification methods for structural model updating are used to provide the entire spectrum of Pareto optimal FE models consistent with the measured modal data. Similarities and differences between the two model updating methods are explored and the advantages of the multi-objective identification methods are emphasized. A significant variability in Pareto optimal models is observed, which is indicative of the uncertainty in the updated FE models. The dependence of the variability of the Pareto models on the information contained in the measured data and the size of model and measurement errors is explored by varying the number of measured modes, number of sensors, FE mesh discretization sizes, and number of model parameters. The effectiveness of the updated Pareto optimal models and their predictive capabilities are assessed. Frequency response functions and fatigue lifetime predictions are used as example of structural performance variables in order to demonstrate the variability in the response predictions that arises from the variability in the Pareto optimal models. A large variability in the response predictions is observed that cannot be ignored in decisions based on updated FE models. The multi-objective optimization method provides the general framework for properly accounting for model uncertainty in model-based response predictions consistent with measured data.
\end{abstract}

\section{INTRODUCTION}

Structural model updating methods [1] have been proposed in the past to reconcile mathematical models, usually discretized finite element (FE) models, with experimental data. The estimate of the optimal model from a parameterized class of models is sensitive to uncertainties that are due to limitations of the mathematical models used to represent the behavior of the real structure, as well as the presence of measurement and processing errors in the data. The number and type of measured modal data used in the reconciling process, as well as the norms used to measure the fit between measured and model predicted characteristics may

\footnotetext{
*Correspondence to: Costas Papadimitriou, Department of Mechanical Engineering, University of Thessaly, Volos 38334, Greece.

${ }^{\dagger}$ E-mail: costasp@uth.gr
} 
also affect the estimate of the optimal model. The optimal structural models resulting from model updating methods can be used for improving the model response and reliability predictions [2], for assessing structural health and identifying structural damage [3-9] and for improving effectiveness of structural control devices [10].

Structural model parameter estimation based on measured modal data [3-6] are often formulated as weighted least-squares estimation problems in which metrics, measuring the residuals between measured and model predicted modal characteristics, are build up into a single weighted residuals (WR) metric formed as a weighted average of the multiple individual metrics using weighting factors. Standard gradient-based optimization techniques are then used to find the optimal values of the structural parameters that minimize the single WRs metric representing an overall measure of fit between measured and model predicted modal characteristics. Due to model error and measurement noise, the results of the optimization are affected by the values assumed for the weighting factors. One of the purposes of this work is to estimate the variability of the optimal values of the model parameters resulting from the variability in the weight values. Such variability clearly defines the uncertainty in the parameter space of the values of the model parameters that should be considered based on the measured data.

To eliminate the need of using weighting factors for assessing the relative importance of each metric in the overall measure of fit, the model updating problem has also been formulated in a multi-objective context [11] that allows the simultaneous minimization of the multiple modal metrics. The multi-objective parameter estimation methodology provides a complete set of multiple Pareto optimal structural models, consistent with the data and the residuals used. The set of Pareto optimal models contains the optimal models obtained by the weighted modal residuals method for any possible values of the weights [12]. However, additional Pareto optimal solutions may exist that do not correspond to a solution of the WRs method for any value of the weights.

Theoretical and computational issues arising in multi-objective identification have been addressed and the correspondence between the multi-objective identification and the WRs identification has been established [11,12]. Emphasis was also given in addressing computational issues associated with solving the resulting multi-objective and single-objective optimization problems. The multi- and single-objective optimization problems are carried out using gradientbased algorithms. The Normal Boundary Intersection (NBI) method [13], in particular, is used as the gradient-based method to solve the multi-objective optimization. Efficient algorithms were introduced for reducing the computational cost involved in estimating the gradients of the objective functions representing the modal residuals. A computationally efficient method for estimating the gradient of the objective functions with respect to the model parameters has been proposed [14] and shown to significantly reduce the computational effort for solving the singleand multi-objective optimization problems. The method exploits Nelson's algorithm [15] for estimating the sensitivity of the eigenproperties with respect to the parameters, requiring the solution of an adjoint eigen-problem and avoiding the explicit estimation of the gradients of the modal frequencies and mode shapes. The computational cost for estimating these gradients is shown to be independent of the number of structural model parameters. The methodology is particularly efficient to system with several number of model parameters and large number of degrees of freedom (DOFs), where repeated gradient evaluations are computationally quite time consuming.

In this work, the applicability and effectiveness of the model updating methods, namely the multi-objective identification method and the WRs method, is explored by updating FE models of a small-scale vehicle frame, using experimentally identified modal data. The vehicle frame also serves as a case study to investigate the variability in the predictions of structural performance indices from these Pareto optimal models. Issues related to estimating unidentifiable solutions [16-18] arising in FE model updating formulations are also addressed. A systematic study is carried out to demonstrate the effect of model error, FE model parameterization, mesh size, number of measured modes, and number of mode shape components on the Pareto optimal models and their variability. It is demonstrated that the updated FE models obtained using measured modal data may vary considerably. Certain similarities and differences between the FE model updating formulations are revealed. The proposed multi-objective identification method 
presents a computationally efficient framework to represent the entire set of updated FE models as well as to investigate the predictions from these models.

This study is organized as follows. Section 2 overviews the two formulations for FE model updating based on modal data, namely the weighted modal residuals method and the multiobjective identification method, establishing the similarities and the differences between the formulations and the computational requirements. Section 3 introduces the small-scale laboratory vehicle frame and gives a brief review of the experimental set-up and the modal identification results. Section 4 presents a series of parametric studies on updating FE models of the vehicle frame and in estimating the whole set of Pareto optimal FE models consistent with the measurements. In Section 5 the variability in the predictions of frequency response functions (FRF) and expected fatigue lifetime, often used as safety index of metallic structures in design, is investigated based on the variability of the Pareto optimal models. Conclusions are summarized in Section 6.

\section{FE MODEL UPDATING METHODS}

\subsection{Modal residuals}

Let $D=\left\{\hat{\omega}_{r}, \hat{\phi}_{r} \in R^{N_{0}}, r=1, \ldots, m\right\}$ be the modal parameters identified by vibration measurements taken from a structure. The modal data in $D$ consist of modal frequencies $\hat{\omega}_{r}$ and mode shape components $\hat{\phi}_{r}$ at $N_{0}$ measured DOFs, where $m$ is the number of observed modes. Consider a parameterized class of linear structural models used to model the dynamic behavior of the structure and let $\underline{\theta} \in R^{N_{\theta}}$ be the set of free structural model parameters to be identified using the identified modal parameters. The objective in a modal-based structural identification methodology is to estimate the values of the parameter set $\underline{\theta}$ so that the modal data $\left\{\omega_{r}(\underline{\theta}), \underline{\phi}_{r}(\underline{\theta}) \in R^{N_{0}}, r=1, \ldots, m\right\}$ predicted by the linear class of models at the corresponding $N_{0}$ measured DOFs best matches the experimentally obtained modal data in $D$. For this, let

$$
\varepsilon_{\omega_{r}}(\underline{\theta})=\frac{\omega_{r}^{2}(\underline{\theta})-\hat{\omega}_{r}^{2}}{\hat{\omega}_{r}^{2}} \quad \text { and } \quad \varepsilon_{\underline{\phi}_{r}}(\underline{\theta})=\frac{\left\|\beta_{r}(\underline{\theta}) \underline{\phi}_{r}(\underline{\theta})-\underline{\hat{\phi}}_{r}\right\|}{\left\|\hat{\hat{\phi}}_{r}\right\|}, \quad r=1, \ldots, m
$$

be the measures of fit or residuals between the measured modal data and the model predicted modal data for the $r$ th modal frequency and mode shape components, respectively, where $\|\underline{z}\|^{2}=\underline{z}^{\mathrm{T}} \underline{z}$ is the usual Euclidean norm, and $\beta_{r}(\underline{\theta})=\underline{\hat{\phi}}_{r}^{\mathrm{T}} \underline{\phi}_{r}(\underline{\theta}) /\left\|\underline{\phi}_{r}(\underline{\theta})\right\|^{2}$ is a normalization constant that guaranties that the measured mode shape $\underline{\hat{\phi}}_{r}$ at the measured DOFs is closest to the model mode shape $\beta_{r}(\underline{\theta}) \underline{\phi}_{r}(\underline{\theta})$ predicted by the particular value of $\underline{\theta}$.

To proceed with the model updating formulation, the measured modal properties are classified next into two groups. The first group contains the modal frequencies while the second group includes the mode shape components for all modes. For each group, a norm is introduced to measure the residuals of the difference between the measured values of the modal properties involved in the group and the corresponding modal values predicted from the model class for a particular value of the parameter set $\underline{\theta}$. For the first group, the measure of fit $I_{1}(\underline{\theta})$ is selected to represent the difference between the measured and the model predicted frequencies for all modes. For the second group, the measure of fit $I_{2}(\theta)$ is selected to represent the difference between the measured and the model predicted mode shape components for all modes. Specifically, the two measures of fit are given by

$$
I_{1}(\underline{\theta})=\sum_{r=1}^{m} \varepsilon_{\omega_{r}}^{2}(\underline{\theta}) \quad \text { and } \quad I_{2}(\underline{\theta})=\sum_{r=1}^{m} \varepsilon_{\underline{\phi}}^{2}(\underline{\theta})
$$

Alternative measures of fit $[3,19-22]$ can easily be accommodated in the proposed formulation. In particular, it is straightforward to show that $I_{2}(\underline{\theta})=\sum_{r-1}^{m}\left(1-\mathrm{MAC}_{r}^{2}\right)$, where $\mathrm{MAC}_{r}=\underline{\phi}_{r}^{T} \underline{\hat{\phi}}_{r} /\left(\left\|\underline{\phi}_{r}\right\|\left\|\underline{\phi}_{r}\right\|\right)$ is the modal assurance criterion between the experimentally 
identified and model predicted mode shapes for the $r$ th mode. Thus, the residuals $I_{2}(\underline{\theta})$ are exactly the same as alternative measures of fit based on MAC values defined in the literature [3].

\subsection{WR method}

The parameter estimation problem is traditionally solved by minimizing the single objective

$$
I(\underline{\theta} ; \underline{w})=w_{1} I_{1}(\underline{\theta})+w_{2} I_{2}(\underline{\theta})
$$

formed by the two objectives $I_{i}(\underline{\theta})$, using the weighting factors $w_{i} \geqslant 0, i=1,2$, with $w_{1}+w_{2}=1$. The objective function $I(\underline{\theta} ; \underline{w})$ represents an overall measure of fit between the measured and the model predicted characteristics. The relative importance of the residual errors in the selection of the optimal model is reflected in the choice of the weights. The results of the identification depend on the weight values used. The optimal solutions for the parameter set $\underline{\theta}$ for a given $\underline{w}$ are denoted by $\hat{\theta}(\underline{w})$.

Conventional WR methods select specific weight values to carry out the parameter estimation. One such choice is $w_{1}=w_{2}=1 / 2$, referred herein as the equally weighted residuals (EWR) method. A more rational choice, however, is to select the weights to be inversely proportional to the values of the modal group residuals obtained from the optimal model associated with these weight values. That is, the optimal values $\hat{w}_{i}$ of the weights are selected to be $\hat{w}_{i}=\alpha_{i} / I_{i}\left(\hat{\theta}_{\text {opt }}\right), i=1,2$, where $\alpha_{1}=m$ and $\alpha_{2}=m N_{0}$ are selected to be the number of modal properties in the two modal groups, and $\hat{\theta}_{\mathrm{opt}} \equiv \underline{\hat{\theta}}(\underline{\hat{w}})$ is the optimal value of the model parameters that minimizes the optimally WRs $I(\underline{\theta} ; \underline{\hat{w}})$ in (3) that corresponds to the weight values $\underline{\hat{w}}$. In this way, modal groups with larger residual errors are given less weight than modal groups with smaller residual errors. This choice guarantees that the optimal model is not biased from measured modal properties that contain significant measurement error or measured modal properties that cannot be well represented by the selected model class. Following such an approach, it has been shown in [12] that the optimal values $\hat{\theta}_{\text {opt }}$ of the structural parameters are obtained by minimizing the sum of the logarithm of the modal residuals

$$
\hat{I}(\underline{\theta})=\sum_{i=1}^{n} \alpha_{i} \ln I_{i}(\underline{\theta})
$$

with respect to the parameter set $\underline{\theta}$ and then computing the optimal weights $\underline{\hat{w}}$ from $\hat{w}_{i}=\alpha_{i} / I_{i}\left(\hat{\theta}_{\text {opt }}\right)$. This method is referred in this work as the optimally WRs (OWR) method. It is worth pointing out that the logarithmic estimator in (4) has also been shown to arise from a Bayesian statistical identification point of view [23].

The single objective identification method is computationally attractive since conventional minimization algorithms can be applied to solve the problem. The optimization of $I(\underline{\theta} ; \underline{w})$ in (3) with respect to $\underline{\theta}$ for a given $\underline{w}$ or the optimization of $\hat{I}(\underline{\theta})$ in (4) with respect to $\underline{\theta}$ can readily be carried out numerically using any available algorithm for optimizing a nonlinear function of several variables. However, these single objective optimization problems are often highly nonconvex that may involve multiple local/global optima. Global optimization algorithms are also available to address cases with multiple local/global solutions [12,24-26].

\subsection{Multi-objective identification (MOI) method}

The problem of identifying the model parameter values that minimize the modal residuals in (2) can also be formulated as a multi-objective optimization problem stated as follows [11]. Find the values of the structural parameter set $\underline{\theta}$ that simultaneously minimizes the objectives

$$
\underline{y}=\underline{I}(\underline{\theta})=\left(I_{1}(\underline{\theta}), I_{2}(\underline{\theta})\right)
$$

where $\underline{\theta}=\left(\theta_{1}, \ldots, \theta_{N_{\theta}}\right) \in \Theta$ is the parameter vector, $\Theta$ is the parameter space, $y=\left(y_{1}, y_{2}\right) \in Y$ is the two-dimensional objective vector and $Y$ is the objective space. For conflicting objectives $I_{1}(\underline{\theta})$ and $I_{2}(\underline{\theta})$ there is no single optimal solution, but rather a set of alternative solutions, known as Pareto optimal solutions, that are optimal in the sense that no other solutions in the parameter space are superior to them when both objectives are considered. The set of objective vectors 
$y=\underline{I}(\underline{\theta})$ corresponding to the set of Pareto optimal solutions $\underline{\theta}$ is called Pareto optimal front. The characteristics of the Pareto solutions are that the residuals in the modal frequencies cannot be improved without deteriorating the residuals in the mode shapes. The multiple Pareto optimal solutions are due to modeling and measurement errors [12].

Introducing a monotonically increasing scalar function $g(x)>0$, with $x>0$ and defining the new objectives $J_{i}(\underline{\theta})=g\left(I_{i}(\underline{\theta})\right), i=1,2$, the Pareto solutions obtained by solving (5) can equivalently be obtained by solving the multi-objective optimization problem

$$
\underline{J}(\underline{\theta})=\left(J_{1}(\underline{\theta}), J_{2}(\underline{\theta})\right)
$$

It can be readily shown that the Pareto solutions are invariant to the selection of $g(x)$. Selecting in particular $g(x)=\sqrt{x / m}$, the new objective functions take the form

$$
J_{1}(\underline{\theta}) \equiv e_{\omega}(\underline{\theta})=\sqrt{\frac{1}{m} \sum_{r=1}^{m} \varepsilon_{\omega_{r}}^{2}(\underline{\theta})} \text { and } J_{2}(\underline{\theta}) \equiv e_{\underline{\phi}}(\underline{\theta})=\sqrt{\frac{1}{m} \sum_{r=1}^{m} \varepsilon_{\underline{\phi}_{r}}^{2}(\underline{\theta})}
$$

representing the average fractional errors between the measured and model predicted eigenvalues (square of the modal frequencies) and mode shapes over all considered modes, respectively. The average fractional errors in the modal frequencies and the mode shapes are more direct measures of the discrepancies between measured and model predicted modal properties. Specifically, in the case of equal modal frequency errors $\varepsilon_{\omega_{1}}(\underline{\theta})=\ldots=\varepsilon_{\omega_{m}}(\underline{\theta})$, the first objective function takes the form $J_{1}(\underline{\theta})=\varepsilon_{\omega_{r}}(\underline{\theta})$ for any mode $r$ and is directly related to the fractional error in each modal frequency. A similar interpretation holds for $J_{2}(\underline{\theta})$.

The set of Pareto optimal solutions can be obtained using available multi-objective optimization algorithms. A very efficient algorithm for solving the multi-objective optimization problem is the Normal-Boundary Intersection (NBI) method [13]. For completeness, the idea of the NBI method is briefly illustrated geometrically in Figure 1 for a two-dimensional objective space. For this, let $\hat{\theta}^{(i)}, i=1,2$, be the global optimal values of the model parameters that minimize the individual objectives $J_{i}(\underline{\theta}), i=1,2$, respectively. The Pareto points $\underline{\hat{J}}^{(i)}=\underline{J}\left(\hat{\theta}^{(i)}\right)$, shown in Figure 1, determine the location of the boundaries of the Pareto front in the objective space. These edge points of the Pareto front are estimated using single-objective optimization algorithms. The utopia point $\underline{J}^{*}=\left[\hat{J}_{1}, \hat{J}_{2}\right]^{\mathrm{T}}$, shown in Figure 1 , is introduced as the point in the objective space with coordinates the individual minima $\hat{J}_{i}=J_{i}\left(\hat{\theta}^{(i)}\right), i=1,2$, of the objectives. Let $\Phi$ be the $2 \times 2$ matrix with the $i$ th column equal to the vector $\hat{J}^{(i)}$. The set of points in the objective space that are convex combinations of $\hat{J}^{(i)}-\hat{J}$, obtained by the points $\left\{\Phi \beta: \beta \in R^{2}, \beta_{1}+\beta_{2}=1, \beta_{i} \geqslant 0\right\}$, is referred to as the Convex Hull of Individual Minima $(\mathrm{C} \overline{\mathrm{H}} \mathrm{M} \overline{\mathrm{M}})$. These points are all points along the line segment AB in Figure 1. The Pareto points lie on the intersection of the boundary $\partial Y$ of the objective space $Y$ and the normal initiating from any point in the CHIM and pointing towards the origin of the objective space.

A point along the Pareto front can be found by solving a single-objective constrained optimization problem. Given the coordinates $\underline{\beta}, \Phi \underline{\beta}$ represents a point on the CHIM and $\Phi \underline{\beta}+t \underline{n}$,

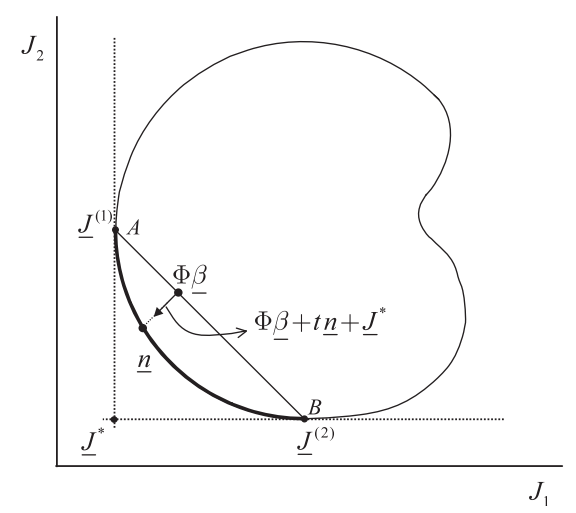

Figure 1. Geometric illustration of the NBI method in a two-dimensional objective space. 
where $t \in R$ and $\underline{n}$ the normal to the CHIM, represents the set of points on the normal to the CHIM at the point $\Phi \beta$. The point of intersection of the normal and the bounbary $\partial Y$, closest to the origin, is the global solution of the commonly referred $\mathrm{NBI}_{\underline{\beta}}$ optimization problem [13]:

$$
\max _{\underline{\theta}, t} t
$$

subject to the equality constraints

$$
\Phi \underline{\beta}+t \underline{n}=\underline{J}(\underline{\theta})-\underline{J}^{*}
$$

This requires the computation of the gradients of the constraints and as a consequence the gradients of the modal residuals $\underline{J}(\underline{\theta})$ involved in (9).

By solving the optimization problems $\mathrm{NBI}_{\beta}$ for various $\beta$ values in the set $\left\{\beta \in R^{2}: \beta_{1}+\beta_{2}=1, \beta_{i} \geqslant 0\right\}$, a pointwise representation of the Pareto front is efficiently constructed. The values of the parameters $\underline{\beta}$ are selected so that an evenly spread set of points along the CHIM is obtained, resulting to an evenly spread set of points along the Pareto front, independently of the scales of the objective functions. This is achieved by selecting the values of $\beta_{2}$ to be uniformly spaced in the interval $[0,1]$ and $\beta_{1}=1-\beta_{2}$. More details about the method can be found in the original paper by Das and Dennis [13].

The NBI optimization method involves the solution of nonlinear constrained optimization problems using available gradient-based constrained optimization algorithms. The NBI uses the gradient information to accelerate convergence to the Pareto front. The computational time required in the NBI method is of the order of the number of points used to represent the Pareto front multiplied by the computational time required to solve a single-objective $\mathrm{NBI}_{\beta}$ problem for computing each point on the Pareto front. The convergence of the NBI method can be greatly accelerated by selecting the Pareto optimal solution obtained from the current $\mathrm{NBI}_{\underline{\beta}}$ problem to be used as starting value for solving the next $\mathrm{NBI}_{\underline{\beta}}$ problem.

\subsection{Multi-objective identification versus WRs}

Certain similarities and differences between the multi-objective identification and the WRs methods are next summarized. The optimal solution to the problem (3) for any value of the weight $\underline{w}$ is one of the Pareto optimal solutions in (5) [12]. Thus, solving a series of single objective optimization problems of the type (3) and varying the values of the weights $w_{i}$ from 0 to 1 , excluding the case for which the values of all weights are simultaneously equal to zero, Pareto optimal solutions are alternatively obtained. However, a severe drawback of generating Pareto optimal solutions by solving the series of weighted single-objective optimization problems by uniformly varying the values of the weights is that this procedure often results in a cluster of points in parts of the Pareto front that fail to provide an adequate representation of the entire Pareto shape. Thus, algorithms dealing directly with the multi-objective optimization problem and generating points that are uniformly spaced along the entire Pareto front should be preferred. The advantages of the NBI algorithm are that it produces evenly spaced points along the Pareto front, even for problems for which the relative scaling of the objectives are vastly different.

Another advantage of the multi-objective identification methodology is that all admissible solutions in the parameter space that simultaneously minimize the two objectives are obtained. In particular, it should be noted that there may exist Pareto optimal solutions that do not arise from the solutions of the single-objective weighted modal residuals problem for any value of the weight vector $\underline{w}$ [12]. Thus, varying the weights in the WRs method does not guarantee the estimation of the entire Pareto front since there may exist extra points on this front that do not correspond to solutions of the single-objective weighted modal residuals problem obtained for all possible values of the weights.

Finally, it should also be pointed out that the Pareto optimal points generated by the WRs norm in (3) can equivalently be obtained by the same WRs norm with $I_{i}(\underline{\theta})$ replaced by $J_{i}(\underline{\theta})=g\left(I_{i}(\underline{\theta})\right)$ for $i=1,2$. The selection of $g(x)$ depends on the user preference in the 
multi-objective identification to evenly space points along the Pareto front in the objective space $\underline{J}=\left(J_{1}, J_{2}\right)$ instead of the original objective space $\underline{I}=\left(I_{1}, I_{2}\right)$.

\section{SMALL-SCALE LABORATORY VEHICLE FRAME AND MODAL IDENTIFICATION}

The model updating methodologies are applied to update FE models of an experimental smallscale vehicle-like body, shown in Figure 2(a). The vehicle structure is housed at the Machine Dynamics Laboratory of the Department of Mechanical Engineering in Aristotle University. It is designed to simulate the frame substructure of a vehicle in a small scale of length $2 \mathrm{~m}$, width $1 \mathrm{~m}$ and height $1.4 \mathrm{~m}$. This small-scale laboratory structure consists of frame substructures (base, connections and top parts in Figure 2(b)) that are welded together. Figure 2(b) presents details of the geometrical dimensions of the frame. The frame structure is made of steel with Young's modulus $E=2.1 \times 10^{11} \mathrm{~N} / \mathrm{m}^{2}$, Poison's ratio $v=0.3$ and density $\rho=7850 \mathrm{~kg} / \mathrm{m}^{3}$. The lower part of the frame is made of hollow rectangular sections with dimensions $100 \mathrm{~mm} \times 50 \mathrm{~mm} \times 3 \mathrm{~mm}$, while the upper part of the frame is also made of hollow rectangular sections with dimensions $50 \mathrm{~mm} \times 50 \mathrm{~mm} \times 2 \mathrm{~mm}$. These two parts are connected together at four points through steel angles with the two faces made of plates with dimensions $300 \mathrm{~mm} \times 100 \mathrm{~mm} \times 10 \mathrm{~mm}$ and $200 \mathrm{~mm} \times 100 \mathrm{~mm} \times 10 \mathrm{~mm}$, as shown in Figure 2(b). More details can be found in [27]. Figure 2(a) also shows an overview of the experimental set up, while the measurement points are indicated in Figure 3. Measurements are collected from 72 locations. The sensor locations have been chosen in such a way so as to gather as much information as possible about the structure's modal response.

Using the available acceleration sensors to measure the vibrations induced by applied impulse forces, the FRF of the measured DOFs are estimated. These FRFs are used to estimate the modal properties using the PolyMAX frequency domain method [28]. The values of the modal frequencies, modal damping ratios and mode shape components were estimated in the 0 to approximately $100 \mathrm{~Hz}$ frequency range. Ten modes were clearly identified with values of modal damping ratios of the order of $0.1-0.5 \%$, which correspond to relatively low damping values. The identified values of the ten modal frequencies and the modal damping ratios are reported in Table I. The identified mode shapes have also been recorded so that they can be used for updating the FE models.

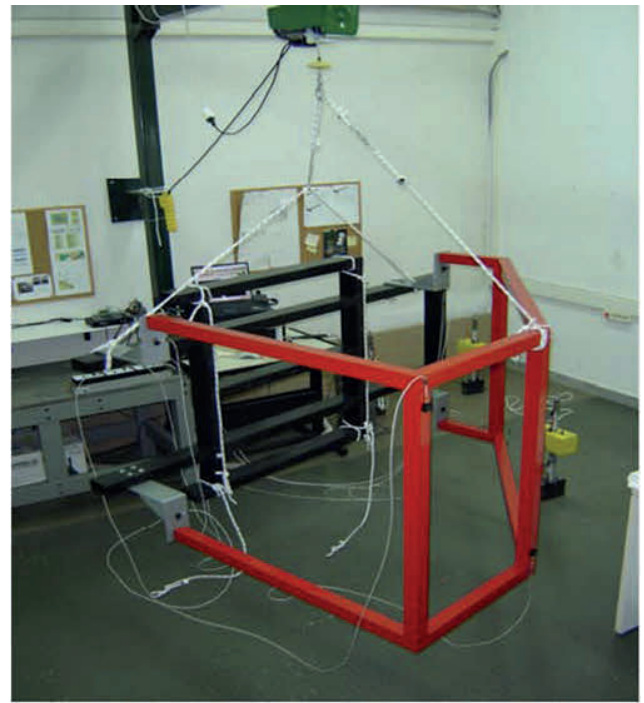

(a)

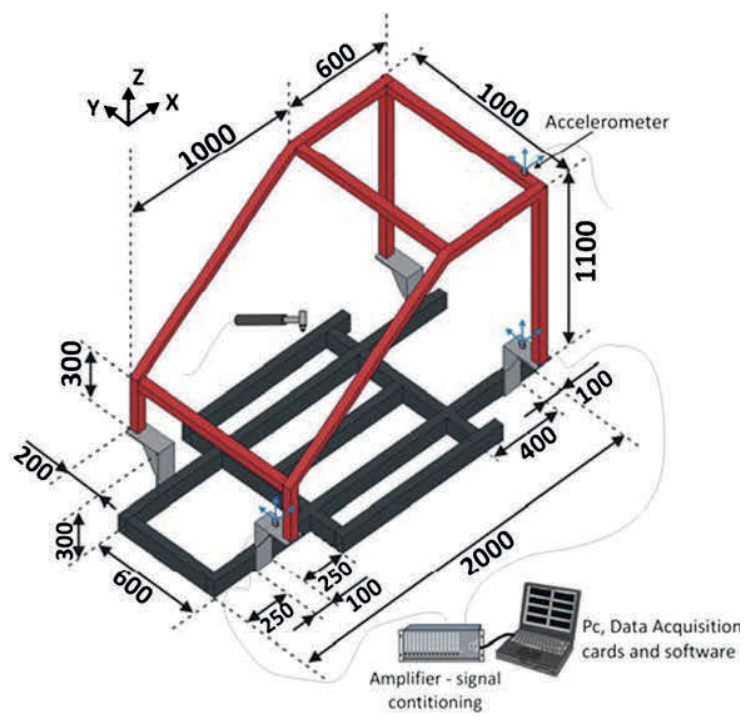

(b)

Figure 2. (a) Scaled vehicle model with experimental set up and (b) Dimensions of the model. 


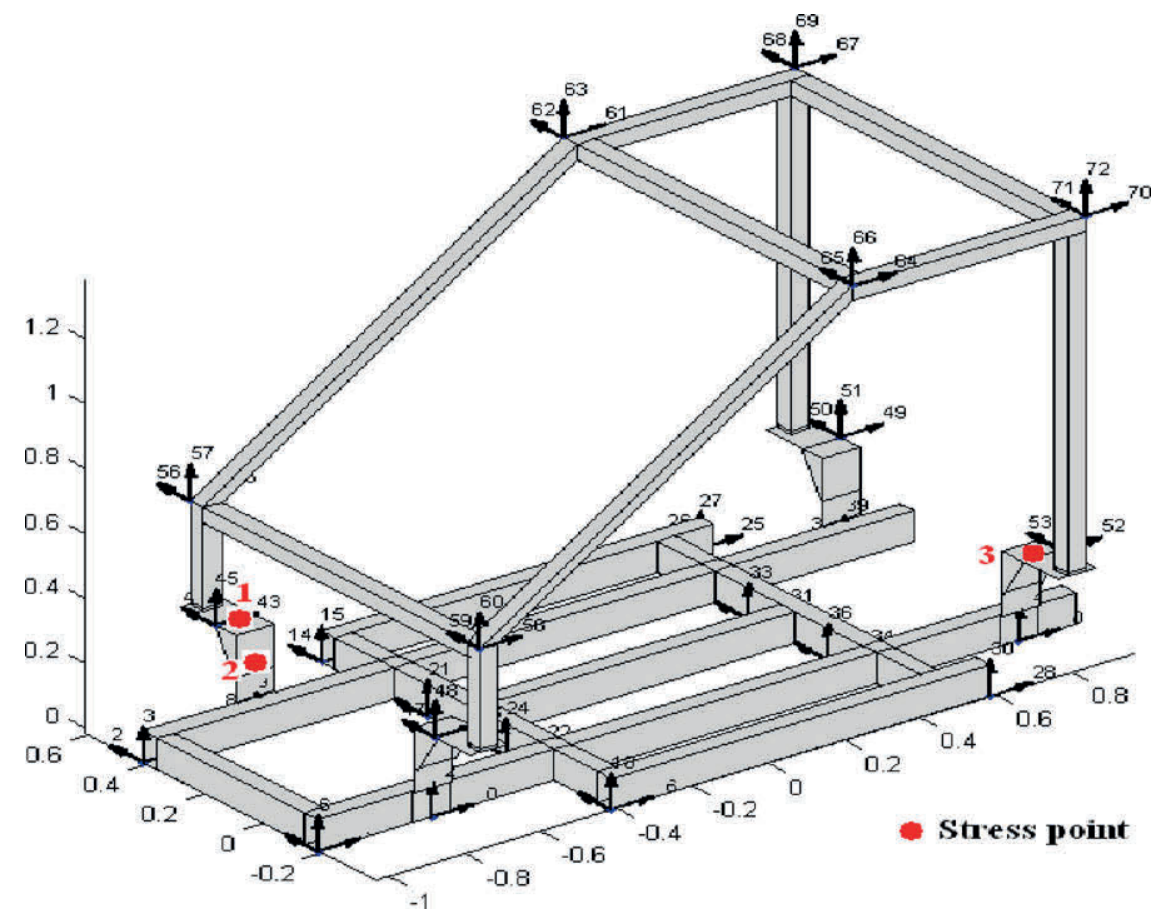

Figure 3. Measurement and stress points on the vehicle frame.

Table I. Identified and nominal FE model predicted modal frequencies and damping ratios, along with percentage errors and MAC values.

\begin{tabular}{|c|c|c|c|c|c|c|c|c|}
\hline \multirow[b]{2}{*}{ Mode } & \multirow{2}{*}{$\begin{array}{l}\text { Identified modal } \\
\text { frequency }(\mathrm{Hz})\end{array}$} & \multirow{2}{*}{$\begin{array}{c}\text { Identified modal } \\
\text { damping ratio }(\%)\end{array}$} & \multicolumn{2}{|c|}{$\begin{array}{l}\text { Nominal FE } \\
\text { predicted modal } \\
\text { frequency }(\mathrm{Hz})\end{array}$} & \multicolumn{2}{|c|}{$\begin{array}{l}\text { Difference between } \\
\text { identified and FE } \\
\text { predicted modal } \\
\text { frequencies }(\%)\end{array}$} & \multicolumn{2}{|c|}{$\begin{array}{l}\text { MAC between } \\
\text { identified and } \\
\text { FE predicted } \\
\text { mode shapes }\end{array}$} \\
\hline & & & Fine & Coarse & Fine & Coarse & Fine & Coarse \\
\hline 1 & 23.21 & 0.48 & 23.23 & 26.67 & 0.09 & 14.89 & 0.971 & 0.972 \\
\hline 2 & 42.12 & 0.36 & 39.13 & 41.48 & -7.11 & -1.52 & 0.977 & 0.976 \\
\hline 3 & 42.50 & 0.23 & 41.61 & 46.21 & -2.10 & 8.73 & 0.979 & 0.955 \\
\hline 4 & 48.28 & 0.22 & 47.29 & 50.94 & -2.03 & 5.53 & 0.973 & 0.953 \\
\hline 5 & 58.16 & 0.20 & 57.57 & 60.60 & -1.01 & 4.20 & 0.951 & 0.951 \\
\hline 6 & 69.04 & 0.23 & 66.20 & 69.94 & -4.11 & 1.31 & 0.975 & 0.970 \\
\hline 7 & 69.47 & 0.17 & 69.05 & 73.48 & -0.60 & 5.77 & 0.950 & 0.945 \\
\hline 8 & 80.04 & 0.17 & 80.44 & 87.26 & 0.50 & 9.02 & 0.950 & 0.940 \\
\hline 9 & 86.14 & 0.16 & 83.25 & 88.14 & -3.36 & 2.31 & 0.968 & 0.959 \\
\hline 10 & 100.24 & 0.11 & 101.60 & 112.97 & 1.36 & 12.69 & 0.979 & 0.852 \\
\hline
\end{tabular}

\section{FE MODEL UPDATING OF THE VEHICLE FRAME}

\subsection{FE models}

Detailed FE models of the vehicle frame were created based on the geometric details and the material properties of the structure. The structure was first designed in CAD environment and then imported in the COMSOL Multiphysics [29] FE modelling environment. The FE models for the vehicle were created using three-dimensional triangular shell FEs to model the whole structure.

A fine mesh model consisting of 15202 FEs and having 45564 DOF was chosen for a detailed modelling of the experimental vehicle. This model is shown in Figure 4(a) and is referred to as 


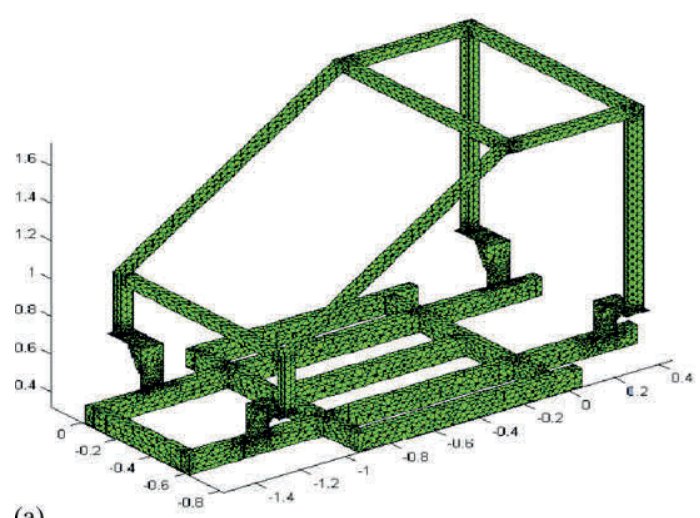

(a)

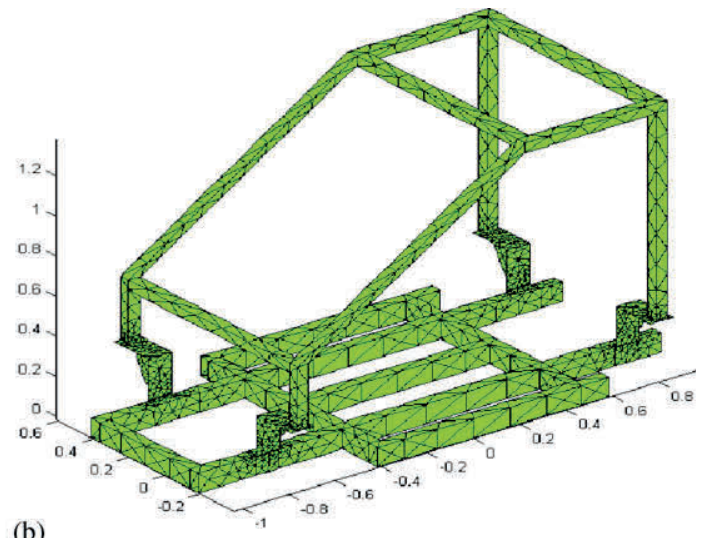

(b)

Figure 4. FE models of the experimental vehicle consisted of (a) 15202 triangular shell elements and 45564 DOF and (b) 2670 triangular shell elements and 8082 DOF.

model $\mathrm{M}_{\mathrm{F}}$. In addition, in order to investigate the sensitivity of the model updating results to model error arising from FE model discretization, a model with a coarser mesh consisting of 2670 FEs and having 8082 DOF was also considered. This model is shown in Figure 4(b) and is referred to as model $M_{C}$. For comparison purposes, Table I lists the values of the modal frequencies predicted by the nominal $\mathrm{FE}$ models $\mathrm{M}_{\mathrm{F}}$ and $\mathrm{M}_{\mathrm{C}}$ along with the percentage error between the experimentally identified modal frequencies and the modal frequencies predicted by the nominal FE models. Also, Table I reports the MAC values between the experimentally identified mode shapes and the mode shapes predicted by the nominal FE models. As expected, the percentage error in the modal frequencies is significantly higher for the coarse model class $\mathrm{M}_{\mathrm{C}}$ than it is for the fine model class $\mathrm{M}_{\mathrm{F}}$. The MAC values for the nominal model $\mathrm{M}_{\mathrm{F}}$ are higher than 0.95, while for the nominal model $\mathrm{M}_{\mathrm{C}}$ are higher than 0.85. The MAC values of the nominal model $M_{C}$ are shown to be less than the MAC values of the nominal model $M_{F}$, verifying that the finer mesh nominal model fits the data better, as should be expected. Ten representative mode shapes predicted by the nominal $\mathrm{FE}$ model $\mathrm{M}_{\mathrm{F}}$ for the ten lowest frequency modes are shown in Figure 5.

\subsection{FE model parameterizations}

Five different parameterizations of the FE model classes $M_{F}$ and $M_{C}$ of the experimental vehicle are introduced in order to demonstrate the applicability of the proposed FE model updating methodologies and point out issues associated with the effect of model error on the Pareto optimal FE models. The parameterized models consisting of three, six, eight, nine and eleven parameters are shown in Figure 6 and are denoted respectively by $M_{3}, M_{6}, M_{8}, M_{9}$ and $M_{11}$. The parameters in the set $\underline{\theta}$ for each model class are stiffness-related parameters and account for the modulus of elasticity of the various parts of the experimental vehicle frame, including the lower and upper parts of the vehicle as well as the connections between these parts. Table II lists for each model class the correspondence of the stiffness-related parameters to the parts of the vehicle that they model. The nominal FE models correspond to values of the model parameters equal to $\theta_{i}=1$ with $i$ ranging from 1 to 11 , depending on the number of parameters in each model class.

\subsection{Pareto front and variability of Pareto optimal FE models}

The three-parameter $\mathrm{FE}$ model class $\mathrm{M}_{3, \mathrm{~F}}$ is updated using the lowest ten identified modal frequencies and mode shapes shown in Table I. The identified mode shapes include components at all 72 sensor locations. This example case is used to demonstrate mainly the applicability of the multi-objective parameter estimation methodology and reveal advantages in relation to the WRs method. 


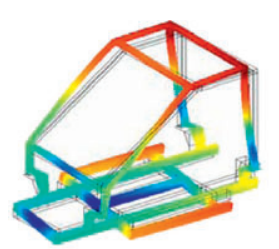

Mode 1: $23.23 \mathrm{~Hz}$
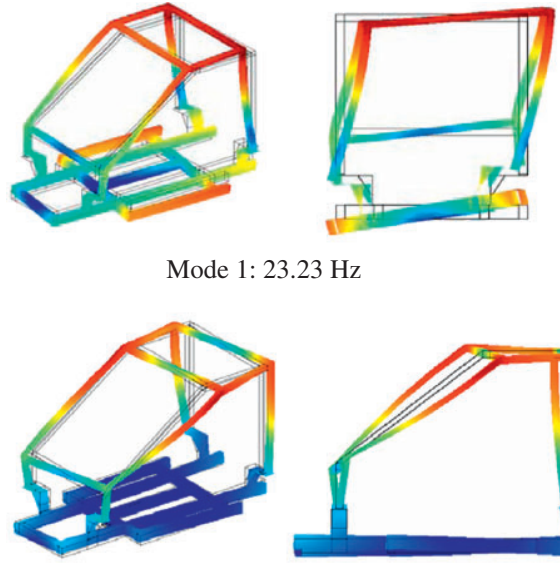

Mode 3: $41.61 \mathrm{~Hz}$

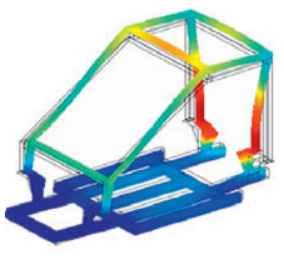

Mode 5: $57.57 \mathrm{~Hz}$
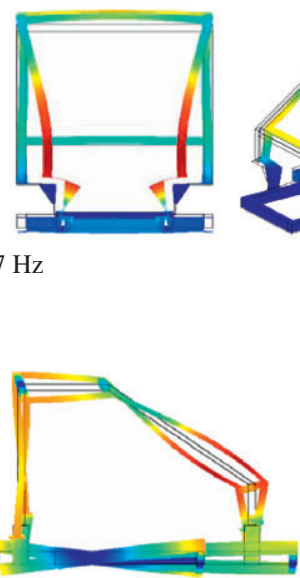

Mode 7: $69.05 \mathrm{~Hz}$

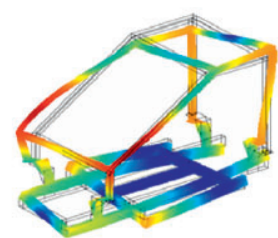

Mode 7:69.05

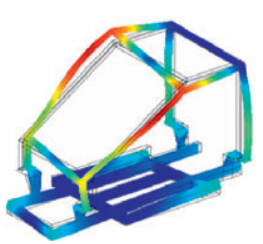

Mode 9: $83.25 \mathrm{~Hz}$

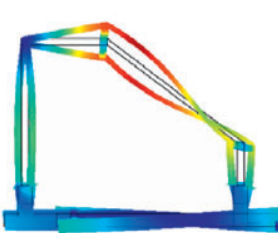

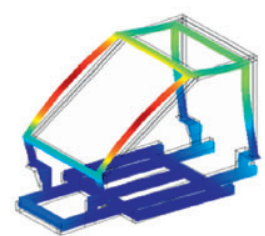

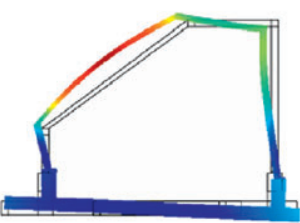

Mode 2: $39.13 \mathrm{~Hz}$

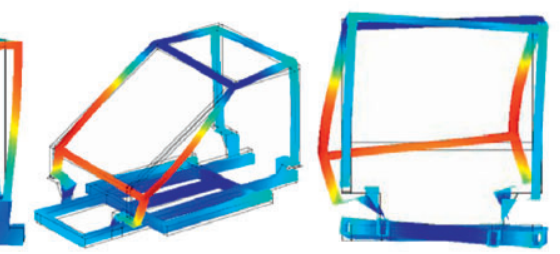

Mode 4: $47.29 \mathrm{~Hz}$
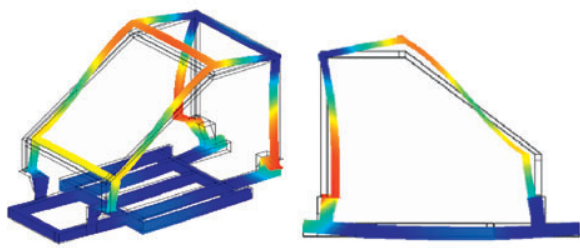

Mode 6: $66.20 \mathrm{~Hz}$

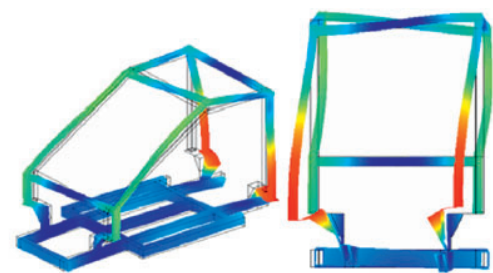

Mode 8: $80.44 \mathrm{~Hz}$

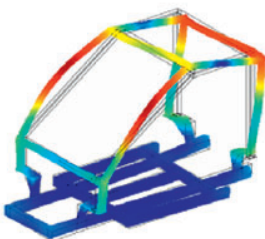

Mode 10: $101.60 \mathrm{~Hz}$

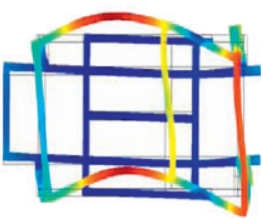

he lowest 10 modes.

The results from the multi-objective parameter estimation methodology are shown in Figure 7. The NBI optimization algorithm was used to estimate 20 Pareto solutions. The Pareto front, giving the Pareto solutions in the two-dimensional objective space, is shown in Figure 7(a). Comparing the Pareto optimal solutions in the objective space, it can be said that there is no Pareto solution that improves the fit in both modal groups simultaneously. Thus, all Pareto solutions correspond to acceptable compromise structural models trading-off the fit in the modal frequencies involved in the first modal group with the fit in the mode shape components involved in the second modal group. The non-zero size of the Pareto front and the non-zero distance of the Pareto front from the origin are due to uncertainties arising from modeling and measurement errors. Specifically, the distance of the Pareto points along the Pareto front from the origin is an indication of how well the model predicted modal characteristics fits the corresponding measured ones. The size of the Pareto front depends on the size of the model error and the sensitivity of the modal properties to the parameter values $\underline{\theta}$ [12]. 

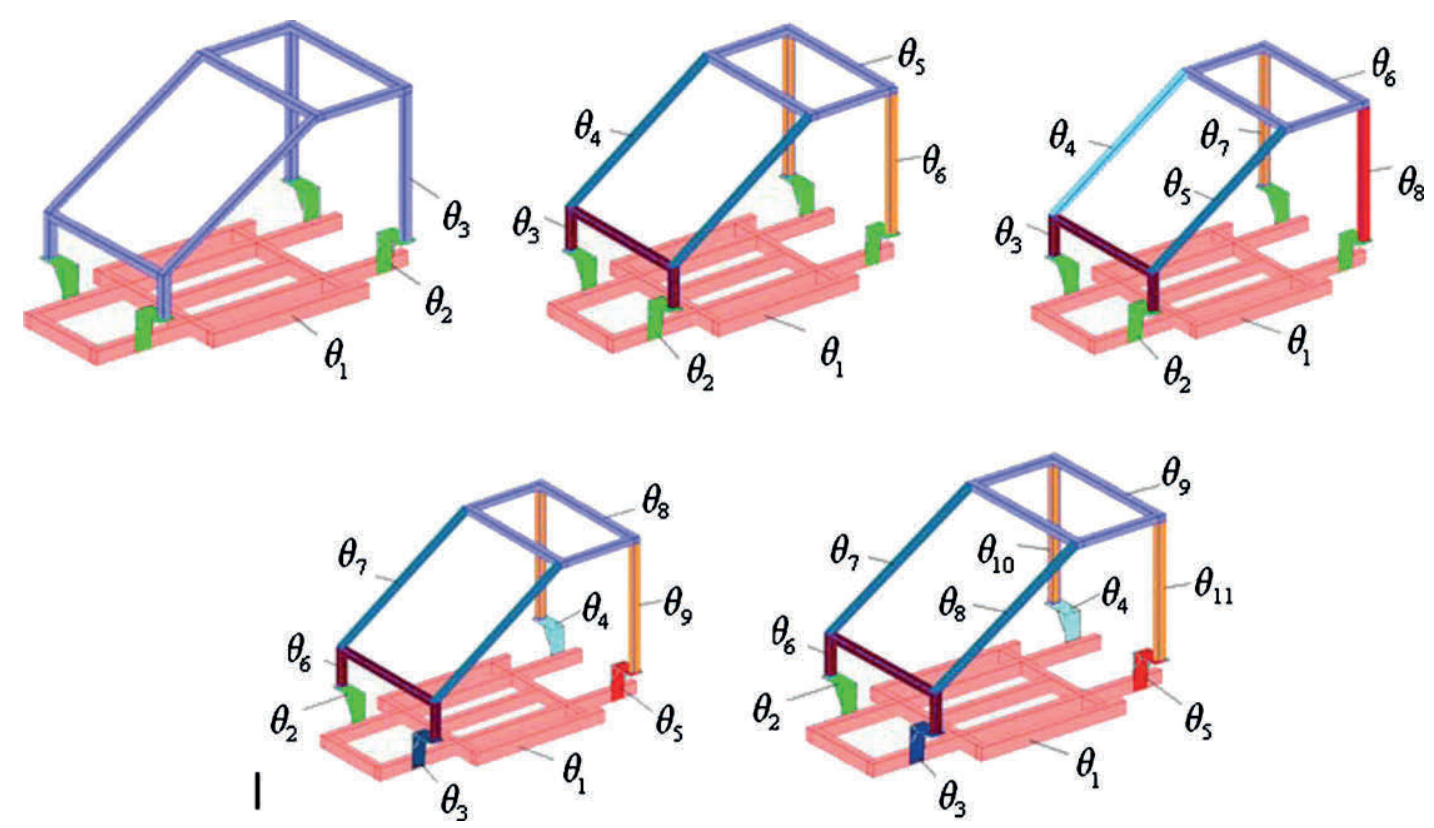

Figure 6. Three, six, eight, nine and eleven parameter model classes of the experimental vehicle frame.

Table II. Definition of model parameters $\theta_{i}$ and percentage variability $\Delta \theta_{i}$ of parameter values for the three, six, eight, nine and eleven parameter model classes.

\begin{tabular}{|c|c|c|c|c|c|c|c|c|c|c|c|c|}
\hline \multirow[b]{2}{*}{ Model class } & \multicolumn{2}{|r|}{ Base } & \multicolumn{2}{|c|}{ Connections } & \multicolumn{2}{|c|}{ Top front (Vertical) } & \multicolumn{2}{|c|}{ Top front (Inclined) } & \multicolumn{2}{|c|}{ Top roof } & \multicolumn{2}{|c|}{ Top rear } \\
\hline & $i$ & $\Delta \theta_{i}(\%)$ & $i$ & $\Delta \theta_{i}(\%)$ & $i$ & $\Delta \theta_{i}(\%)$ & $i$ & $\Delta \theta_{i}(\%)$ & $i$ & $\Delta \theta_{i}(\%)$ & $i$ & $\Delta \theta_{i}(\%)$ \\
\hline $\mathrm{M}_{3, \mathrm{~F}}$ & 1 & 10 & 2 & 22 & 3 & 6 & 3 & 6 & 3 & 6 & 3 & 6 \\
\hline $\mathrm{M}_{6, \mathrm{~F}}$ & 1 & 9 & 2 & 5 & 3 & 29 & 4 & 19 & 5 & 7 & 6 & 17 \\
\hline \multirow[t]{2}{*}{$\mathrm{M}_{8, \mathrm{~F}}$} & 1 & 9 & 2 & 5 & 3 & 29 & 4 & 24 & 6 & 7 & 7 & 20 \\
\hline & & & & & & & 5 & 17 & & & 8 & 20 \\
\hline \multirow[t]{4}{*}{$\mathrm{M}_{9, \mathrm{~F}}$} & 1 & 10 & 2 & 19 & 6 & 34 & 7 & 7 & 8 & 5 & 9 & 4 \\
\hline & & & 3 & 14 & & & & & & & & \\
\hline & & & 4 & 7 & & & & & & & & \\
\hline & & & 5 & 3 & & & & & & & & \\
\hline \multirow[t]{4}{*}{$\mathrm{M}_{11, \mathrm{~F}}$} & 1 & 10 & 2 & 17 & 6 & 37 & 7 & 9 & 9 & 6 & 10 & 13 \\
\hline & & & 3 & 10 & & & 8 & 9 & & & 11 & 5 \\
\hline & & & 4 & 18 & & & & & & & & \\
\hline & & & 5 & 8 & & & & & & & & \\
\hline
\end{tabular}

Figure 7(b-d) shows the corresponding Pareto optimal solutions in the three-dimensional parameter space. Specifically, these figures show the projection of the Pareto solutions in the two-dimensional parameter spaces $\left(\theta_{1}, \theta_{2}\right),\left(\theta_{1}, \theta_{3}\right)$ and $\left(\theta_{2}, \theta_{3}\right)$. The Pareto optimal solutions, quantifying the uncertainties in the model parameter values consistent with measured data, are concentrated along a one-dimensional manifold in the three-dimensional parameter space. It is observed that a wide variety of Pareto optimal solutions are obtained, which are consistent with the measured data and the objective functions used. The variability in the values of the model parameters, computed as the percentage difference between the highest and lowest value relative to the average value of each parameter, are of the order of 10,22 and $6 \%$ for $\theta_{1}, \theta_{2}$ and $\theta_{3}$, respectively. This relatively large variability in the Pareto optimal solutions affects the variability in the predictions from the Pareto optimal models. Such prediction variability is studied in Section 5.

The optimal solutions computed using the EWR and the optimally weighted residuals (OWR) techniques are also shown in Figure 7. As expected, these solutions are points along the 

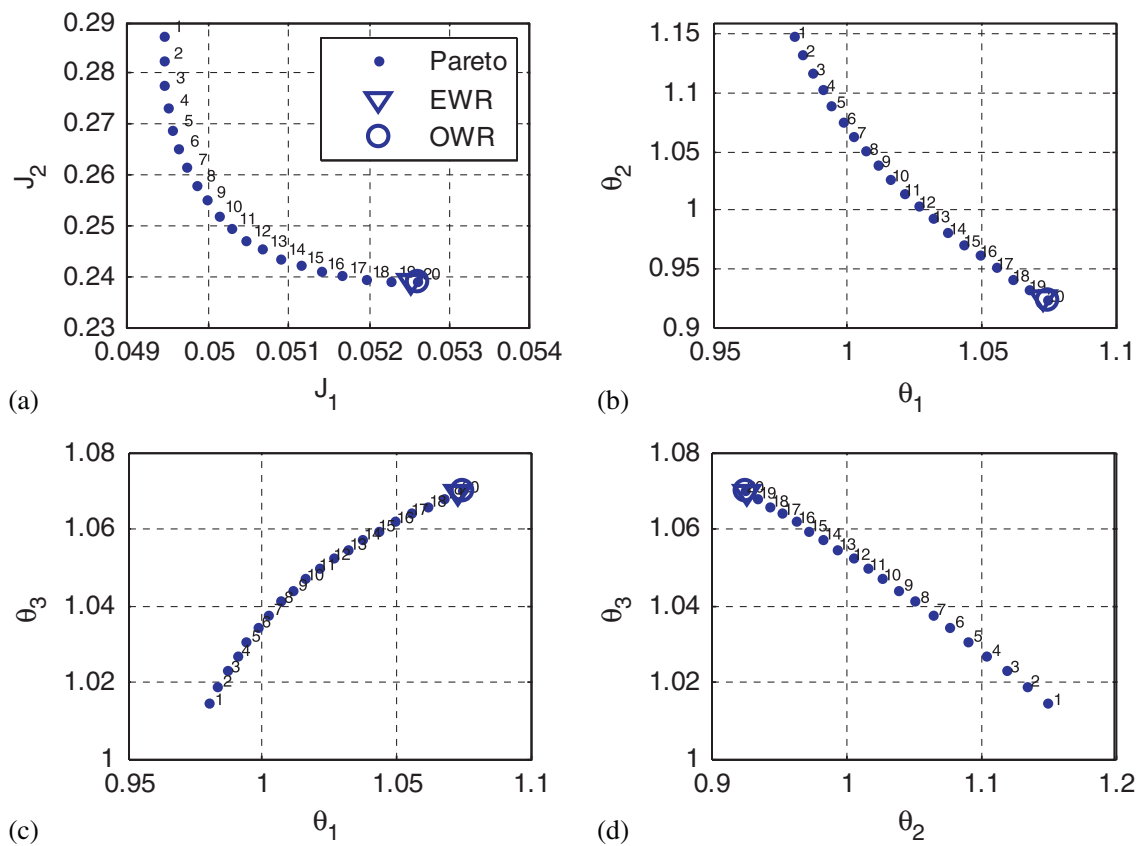

Figure 7. Pareto front and Pareto optimal solutions for the three-parameter model classes in the (a) objective space and (b-d) parameter space.

Pareto front. In fact, in this example case, they have been estimated to be very close to each other in the objective and parameter space. The conventional optimal solution obtained by the EWR method is demonstrated to be in the vicinity of the Pareto solution 20, which is located very close to the right identifiable end of the Pareto front. Comparing the entire set of Pareto optimal solutions and using the proximity of the EWR solution to the Pareto solution 20, it can be said that the EWR optimal solution for this example case almost coincides with the solution that one would obtain by minimizing the residuals in the mode shapes only, ignoring the fit in the modal frequencies. Exactly the same conclusion can be reached for the OWR optimal solution. However, the complete set of Pareto optimal solutions clearly indicate that there exist alternative optimal solutions that take into account the fit in the modal frequencies as well. Such solutions define the uncertainty sub-domain in the parameter space over which the parameters can take values based on the measured data.

\subsection{Unidentifiability issues}

It should be noted that in the results presented in Figure 7 for the model class $M_{3, F}$, only the useful 'identifiable part' of the Pareto front is shown. It will be demonstrated in this section that the multi-objective identification method is efficient in also estimating the unidentifiable solutions often arising in model updating techniques.

First, in Figure 8(a) is shown the extended set of solutions that were actually computed by the application of the NBI algorithm for the model class $M_{3, F}$. Specifically, it is observed that there is a flat part of the extended set of solutions at the lower right edge of Figure 8(a). This is due to the unidentifiability problems [12,16-18] encountered in estimating the optimal model corresponding to the right edge point of the Pareto front. In this case, the right edge point of the Pareto front is obtained by optimizing the function $J_{2}(\underline{\theta})$. It turns out that there is a lower dimensional manifold in the three-dimensional parameter space, shown in the two-dimensional projection $\left(\theta_{1}, \theta_{2}\right)$ in Figure 8(b) to extend from point 12 to point 20, that give the same optimum for $J_{2}(\underline{\theta})$ as it can be seen in Figure 8(a). Depending on the starting values of the parameter set $\underline{\theta}$, the gradient-based algorithm for optimizing $J_{2}(\underline{\theta})$ converges to one of the infinite number of optimal models in this lower dimensional sub-manifold. 

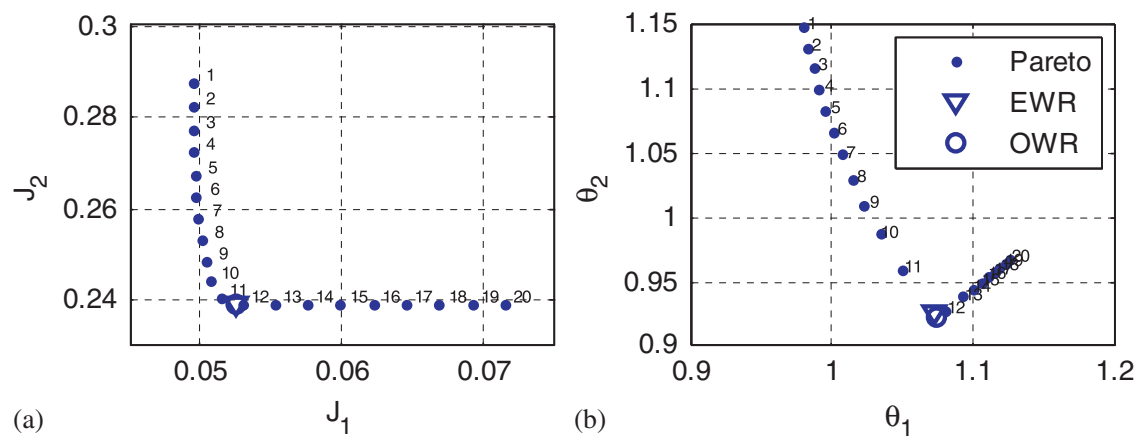

Figure 8. Pareto front and Pareto optimal solutions for the three-parameter model class $\mathbf{M}_{3, \mathrm{~F}}$ in (a) objective space and (b) projection in two-dimensional parameter space $\left(\theta_{1}, \theta_{2}\right)$.

Such unidentifiable manifolds have been reported in previous studies [12,16-18] using simplified models of structures that involve a small number of DOFs and simulated data. This case study demonstrates that unidentifiability issues arise in updating FE models with a large number of DOFs, build for realistic structures and using real measurements. As it is noted in Figure $8(\mathrm{a})$, the unidentifiable solutions corresponding to the flat horizontal portion (points 12-20) in the objective space and the associated manifold in Figure 8(b) are readily estimated by the NBI method. From the engineering point of view, the most important point from this flat portion is the most left point 12 in Figure 8(a) since all other points in the flat portion deteriorate the fit in the objective function $J_{1}(\underline{\theta})$ without altering the fit in $J_{2}(\underline{\theta})$. Alternatively, using Pareto terminology, all points from 13 to 20 are dominated by point 12 and therefore they are not considered as Pareto solutions. For convenience, the unidentifiable solutions estimated by the NBI algorithm will be said to form the unidentifiable part of the Pareto front. Unidentifiability problems usually arise also in the left edge of the Pareto front when the number of modes to be fitted is less than the number of the parameters to be estimated. Examples will be presented in Section 4.8.

Note that in order to generate points only on the identifiable portion of the Pareto front for pre-selected number of points on it (e.g. 20 points as shown in Figure 7(a)), the analyst should repeat the application of the NBI algorithm with edge points of the Pareto front selected to be the points 1 and 12 in Figure 8.

Concluding, the present multi-objective identification methodology is capable of estimating and graphically representing portions of the unidentifiable solutions as well as identifying the meaningful, from the Pareto terminology point of view, identifiable part of the Pareto front and the Pareto optimal solutions.

\subsection{Comparison between $M O I$ and $W R$ methods}

For comparison purposes, the WR method was also used to obtain the Pareto front by uniformly varying the weights in (3) from 0 to 1 and minimizing the objective function in (3) with $I_{i}(\underline{\theta})$ replaced by $J_{i}(\underline{\theta})$ defined in (7). Specifically, dividing the interval $[0,1]$ for the weight $w_{2}$ into equally spaced sub-intervals using a step $\Delta w$, selecting the values of $w_{1}$ to satisfy $w_{1}+w_{2}=1$ and estimating the optimal solutions for the $1 / \Delta w$ pairs of $\left(w_{1}, w_{2}\right)$ values, the Pareto front and the Pareto solutions are obtained and shown in Figure 9(a, b) for model class $\mathrm{M}_{3, \mathrm{~F}}$ for $\Delta w=0.05$ $(1 / \Delta w=20)$. It can be seen from Figure 9 that varying uniformly the weight values and computing the Pareto points using the WR method does not produce uniformly distributed points along the Pareto front. Instead, it yields a cluster of 19 WR points concentrated between the NBI-based Pareto solutions 16 to 20. As a consequence, the part of the Pareto front between Pareto solutions 2 to 15 is misrepresented since it is completely missed. Therefore, a clear advantage of the multi-objective identification versus the WR method is that it represents the Pareto front by a number of points that are uniformly distributed in the objective and the parameter space. 

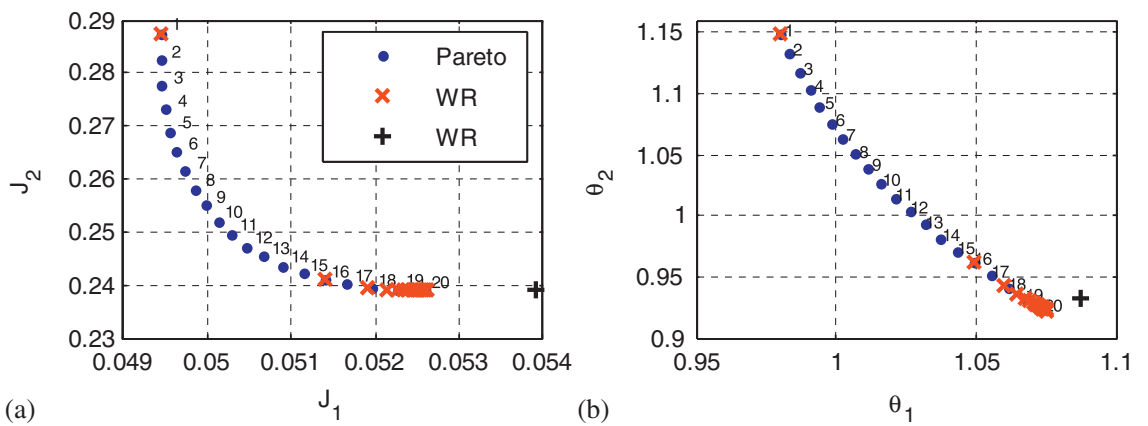

Figure 9. Comparison of Pareto optimal solutions obtained from the NBI algorithm and the WR method by uniformly varying the weight values.

It should also be noted that using the WR method, a series of $i=1, \ldots, 1 / \Delta w$ single-objective optimization problems are solved to compute the points along the Pareto front in Figure 9(a). To accelerate convergence and avoid, to the extent possible, premature convergence to a local optimum, the starting values of the parameters used to solve the $i$ optimization problem are selected to be the optimal parameter values obtained from the $i-1$ optimization problem. Starting from the left edge point 1 of the Pareto front, the iterative process converges for the last value of $i=1 / \Delta w$ to the right edge point 20 of the identifiable part of the Pareto front. For small enough values of $\Delta w$ so that the initial estimate of the previous iteration is close to the optimum estimate of the last iteration, the most probable Pareto point is the point 20, corresponding to optimizing the objective $J_{2}(\underline{\theta})$, while points along the non-identifiable part of the Pareto front (points 13-20 in Figure 8) are not likely to be generated in the last iteration $i=1 / \Delta w$. However, for initial estimates that are far away from the optimum Pareto point 20, such a procedure will fail to give the Pareto point 20 and will converge to one of the models along the unidentifiable part of the Pareto front.

Consider next the estimation of Pareto points using the WR method in the reverse order, starting from the right edge and moving to the left edge. The Pareto point 20, which constitute the first solution at the right edge, will be missed due to the fact that the initial estimate is arbitrary and the algorithm will converge to a point along the unidentifiable part of the Pareto front. The location of the single point on the non-identifiable part of the Pareto front depends on the starting values used to solve the first optimization problem corresponding to optimizing the objective $J_{2}(\underline{\theta})$. For the specific example, the value of $\underline{\theta}=\underline{1}$, used as the initial estimate, resulted in the point denoted by ' + ' in Figure 9(a). As one moves for the last value of $i=1 / \Delta w$ to the left edge point of the Pareto front corresponding to optimizing the objective $J_{1}(\underline{\theta})$, there is no guarantee that it will reach the Pareto point 1 due to the fact that the initial estimate for computing the last point 1, taken as the optimal estimate of the previous iteration (close to Pareto point 16), may be far away from the optimum estimate (Pareto point 1) and the algorithm may prematurely converge to a local optimum. Extra difficulties will arise if the problem of estimating the model parameters based only on the modal frequency residuals is unidentifiable. Then, the solution will converge to a point along the unidentifiable part of the Pareto front. Thus, it becomes clear that varying the weight values in the WR method, important Pareto points such as the boundary points can be missed, especially in the case where unidentifiable solutions exist. On the other hand, the NBI algorithm is computationally very efficient to identify the useful part of the Pareto front even if unidentifiable solutions exist for the two edge points.

\subsection{Effect of number of parameters on Pareto front and optimal FE models}

The Pareto fronts for the case of the six, eight, nine and eleven parameters and the fine mesh model $\mathrm{M}_{\mathrm{F}}$ are shown in Figure 10. Comparing these results with the results in Figure 7(a), obtained for the case of the three-parameter model, it can be concluded that the model class 


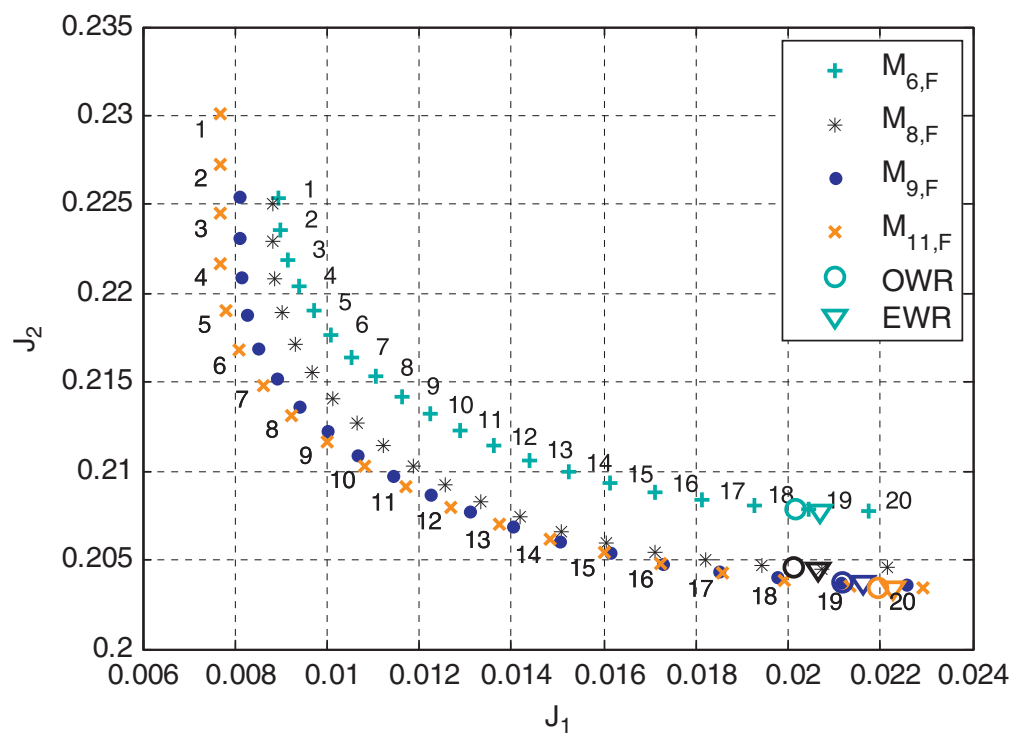

Figure 10. Pareto fronts for the six, eight, nine and eleven parameter model classes.

$\mathrm{M}_{3, \mathrm{~F}}$ with three parameters gives a significantly worse fit to the experimental results considering both objectives $J_{1}$ and $J_{2}$. Specifically, the variability in the average fractional error $e_{\omega}=J_{1}$ in the modal frequencies drops from values ranging from 0.049 to 0.053 for the model class $M_{3, F}$ (Figure 7(a)) to values ranging from 0.008 to 0.022 (Figure 10) for the model classes $M_{6, F}$ to $\mathrm{M}_{11, \mathrm{~F}}$. Similarly, the variability in the average fractional error $\varepsilon_{\phi}=J_{2}$ in the mode shapes drops from values ranging from approximately $0.24-0.29$ for the model class $M_{3, F}$ (Figure 7 (a)) to values ranging from 0.20 to 0.23 (Figure 10) for the model classes $M_{6, F}$ to $M_{11, F}$. Using Pareto terminology, it can be said that all Pareto points for model classes $M_{6, F}$ to $M_{11, F}$ dominate all Pareto points for model class $\mathrm{M}_{3, \mathrm{~F}}$.

In Figure 10 it is observed that the Pareto fronts as the number of model parameters is increased from 6 to 11 move closer to the origin and they do not intersect, indicating that the fit gets better when the number of parameter increases. It should be made clear that this trend occurs due to the fact that the model with a fixed number of parameters, say 9 , contains the model with fewer parameters, say 6 and 3, in the sense that the nine-parameter model can make the same predictions as the 3- and 6-parameter model classes do, but the nine-parameter model has more parameters and thus more freedom to provide a better fit to the data. However, the improvement obtained by increasing the number of parameters from six to eleven is not as significant as the improvement obtained by increasing the number of parameters from three to six.

The corresponding Pareto optimal solutions for the six, eight, nine and eleven parameter model classes are shown in Figure $11(\mathrm{a}-\mathrm{d})$ as a function of the Pareto points. The variability $\Delta \theta_{i}=200\left|\theta_{i, \text { max }}-\theta_{i, \text { min }}\right| /\left(\theta_{i, \text { max }}+\theta_{i, \text { min }}\right)$ in the values of the model parameters, computed as the percentage difference between the highest value $\theta_{i, \max }$ and lowest value $\theta_{i, \min }$ relative to the average value $\left(\theta_{i, \max }+\theta_{i, \min }\right) / 2$ for each parameter $\theta_{i}$, is shown in Table II for the different model classes, including the three-parameter model class $\mathrm{M}_{3, \mathrm{~F}}$. It should be noted that a significant variability in the values of the model parameters is observed for all model classes. The highest variability of the order of $29-37 \%$ is observed for the stiffness of the members located at the front part (Figure 6) of the vehicle. The lowest variability of the order of 5-7\% is consistently observed in the stiffness of the horizontal members located at the roof of the top part of the vehicle model. Low variability is also observed in the stiffness of some of the connections between the lower and upper part of the vehicle.

The percentage error between the experimentally identified values of the modal frequencies and the values of the modal frequencies predicted by the nominal model, the EWR model, the OWR model and the Pareto optimal models 1,10 and 20 are reported as a function of the mode 

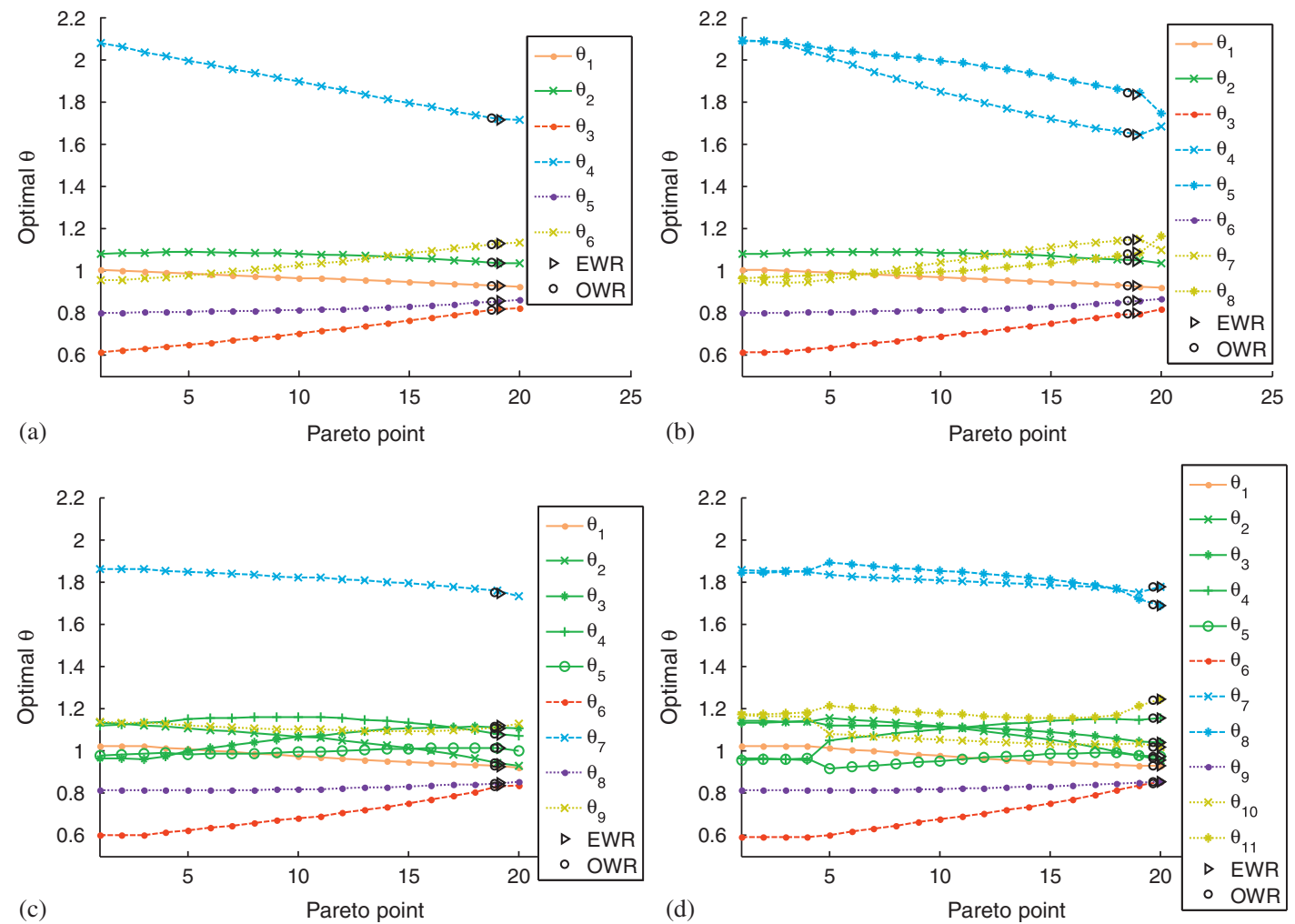

Figure 11. Pareto optimal solutions for the (a) six; (b) eight; (c) nine; and (d) eleven-parameter model classes.

number in Figure 12(a, c, e) for the three, six and nine-parameter model classes, respectively. Similar results for the MAC values are reported in Figure 12(b, d, f). It is observed that the Pareto model 1 provides the best fit to the modal frequencies in the expense of deteriorating the fit in the MAC values to values significantly smaller than one. The Pareto model 20 improves the MAC values to values closer to one in the expense of deteriorating the fit in the modal frequencies. The Pareto model 10 trades-off the fit in the modal frequencies with the fit in the MAC values.

Comparing Figure 12(a, c, e), it can be said that the modal frequency errors for all Pareto optimal solutions for the six and the nine-parameter models do not exceed the values of $2 \%$, which are significantly smaller than the errors for the three-parameter model class estimated to be as high as $6 \%$. Comparing Figure 12(b, d, f), the worst MAC values for the six and the nineparameter model classes are as low as 0.95 , which should be compared to the worst MAC value of 0.91 for the three-parameter model class. The MAC values for the Pareto solution 20 also tend to increase overall in relation to the MAC values of the Pareto solution 1. Comparing the modal frequency errors and the MAC values between the six and the nine-parameter model, it can be seen that the nine-parameter model class provides a slight improvement in the fit of the modal characteristics with respect to the six-parameter model class.

Concluding, based on the fit in the modal frequencies and the MAC values for each mode, it can be said that the six and nine-parameter models give a much better fit to the experimentally identified modal data than the three-parameter model class. The nine-parameter model class gives overall a slightly better fit to the data than the six-parameter model class.

\subsection{Effect of FE mesh size on Pareto front and optimal FE models}

The effect of model error on the FE model updating results is next investigated. The size of model error is controlled by changing the mesh size in the FE discretization. Specifically, model 

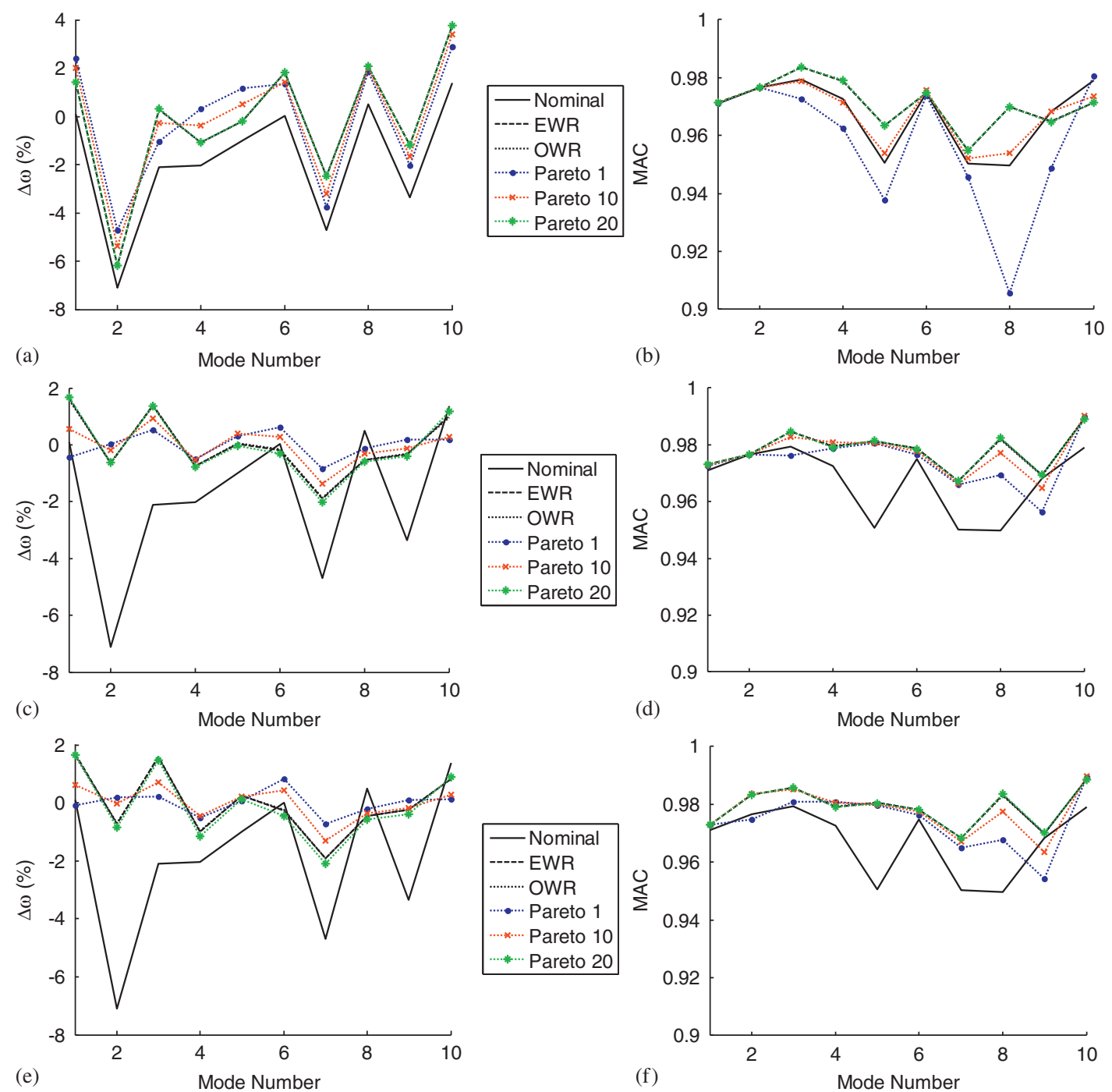

(d)

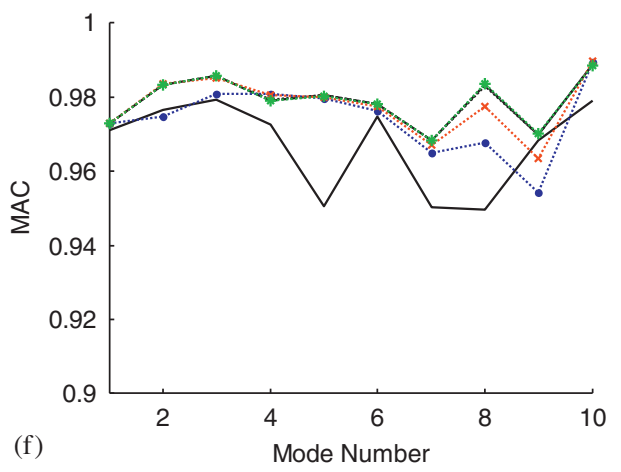

Figure 12. (a, c, e) Percentage error between experimental and model predicted modal frequencies. (b, d, f) MAC values between experimental and model predicted mode shapes. (a, b) $\mathbf{M}_{3, \mathrm{~F}}$, (c, d) $\mathrm{M}_{6, \mathrm{~F}}$, (e, f) $\mathrm{M}_{9, \mathrm{~F}}$.

updating results are obtained for the model classes $\mathrm{M}_{\mathrm{F}}$ and $\mathrm{M}_{\mathrm{C}}$ corresponding to fine and coarse meshes, respectively, as shown in Figure 4. The Pareto fronts obtained for the three, six and nine-parameter models are shown in Figure 13(a) for both fine and coarse mesh model classes $\mathrm{M}_{\mathrm{F}}$ and $\mathrm{M}_{\mathrm{C}}$.

Comparing the Pareto fronts for the fine and coarse mesh model classes with same number of parameters, it is observed that the Pareto front corresponding to the fine mesh model class is consistently closer to the origin of the objective space than the Pareto front corresponding to the coarse mesh model class. This is due to the fact that the fine mesh model class is a higher fidelity model, involving less model error, and thus is expected to fit the measured modal data better, giving smaller residual errors in both modal frequencies and mode shapes.

It is worth noting that for the high fidelity fine-mesh model classes, the Pareto front moves towards the origin as the number of parameters increases from three to nine. A similar trend is also observed for the less accurate coarse-mesh model class. However, the size of the Pareto front in the coarse-mesh model class is significantly increased as the number of parameters increases, indicating that there is a larger variability of the Pareto optimal models for the less accurate coarse-mesh model class than the variability of the Pareto optimal models for the fine 

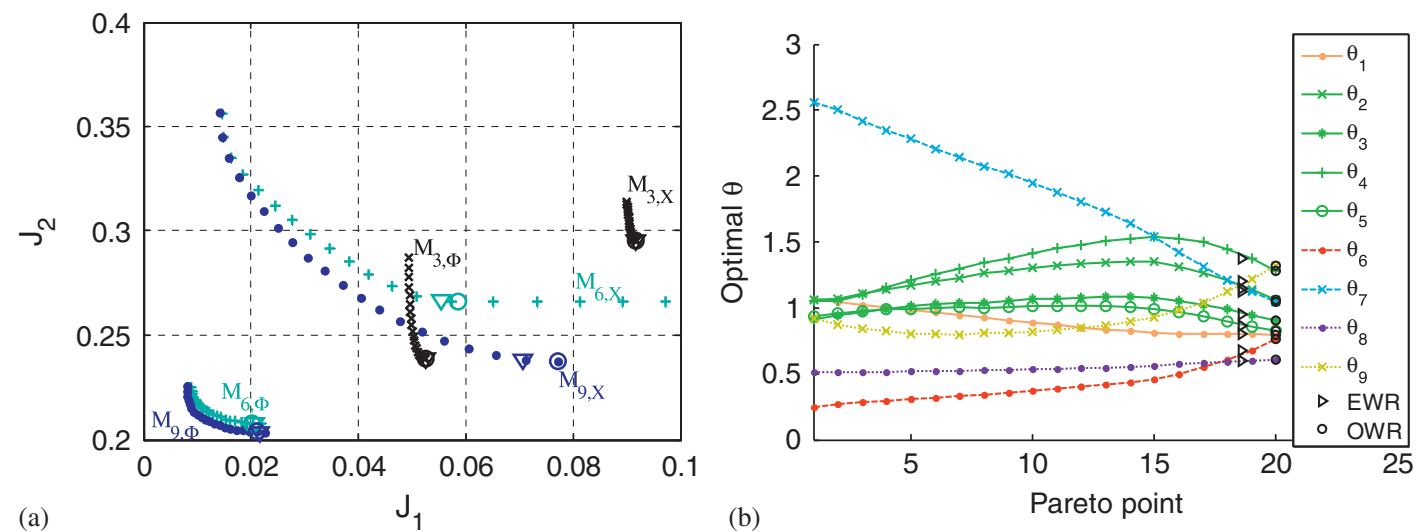

Figure 13. (a) Pareto front for the fine- and coarse-mesh model classes $M_{F}$ and $M_{C}$, involving three, six and nine parameters and (b) Pareto optimal solutions for the nine-parameter coarse-mesh model class $\mathbf{M}_{9, \mathrm{C}}$.

mesh model class. Higher fidelity model classes are expected in general to give smaller variability as the number of free parameters increases.

An interesting observation that can also be made by comparing the results for the threeparameter fine mesh model class $M_{3, F}$ with the nine-parameter coarse mesh model class $M_{9, \mathrm{C}}$ is that the Pareto solutions from the coarse model class $M_{9, C}$ do not provide a better fit simultaneously in both the modal frequencies and mode shapes than the Pareto solutions from the fine mesh model class $M_{3, F}$. This is an example demonstrating that increasing the number of parameters in a model class does not necessarily result in a better fit than a higher fidelity model class involving fewer number of parameters.

The corresponding variability of the Pareto optimal model parameters for the nine-parameter coarse model class $M_{9, C}$ is shown in Figure 13(b). Comparing with Figure 11(c) for the nineparameter fine mesh model class $\mathrm{M}_{9, \mathrm{~F}}$, a significantly larger variability is observed for the values of the parameters of the coarse mesh model class $\mathrm{M}_{9, \mathrm{C}}$. This is especially evident for parameters $\theta_{6}$ and $\theta_{7}$ related to the stiffness of the top front vertical and inclined members, respectively. In particular, the variability of the parameter $\theta_{7}$ along the Pareto solutions ranges from 2.5 to 1 , which is of the order of $130 \%$. In contrast, the variability of the same parameter $\theta_{7}$ for the higher fidelity model class $\mathrm{M}_{9, \mathrm{~F}}$ is shown in Figure 11(c) to range from 1.85 to 1.75 , which is of the order of 5\%. The results in Figures 11(c) and 13(b) suggest that higher fidelity model classes also tend to reduce the variability of the Pareto optimal solutions. Such variabilities are expected to affect the variabilities of the system performance predicted using the entire set of Pareto optimal models.

\subsection{Effect of the number of modes on Pareto front and optimal FE models}

The nine-parameter FE model class $\mathrm{M}_{9, \mathrm{~F}}$ corresponding to the fine mesh is next updated using the five and ten lowest identified modes. The Pareto fronts are shown in Figure 14(a) and are denoted by $M_{9, F}-5$ and $M_{9, F}-10$ for the cases of 5 and 10 modes, respectively. As expected, the modal residuals computed from the Pareto optimal points for the case of 5 modes are smaller than the modal residuals for the case of 10 modes. This is clearly evident by the closer proximity of the Pareto front computed for 5 modes to the origin in the objective space. For the $\mathrm{M}_{9, \mathrm{~F}}-5$ case, it can also be observed that the part of the Pareto front from 1 to 7 constitutes the unidentifiable part of the Pareto front that arises by minimizing the modal frequency residuals $J_{1}(\underline{\theta})$. This unidentifiability is expected since the number of nine free parameters in the model class is higher than the number of five modal frequencies used in this model updating case and so there is an infinite number of solutions that can perfectly fit the five modal frequencies. Also, it should be noted that the modal frequency residuals are zero for Pareto points 1 to 7 , indicating that the fit is perfect. Similarly, the Pareto points 17 to 20 constitute the unidentifiable 

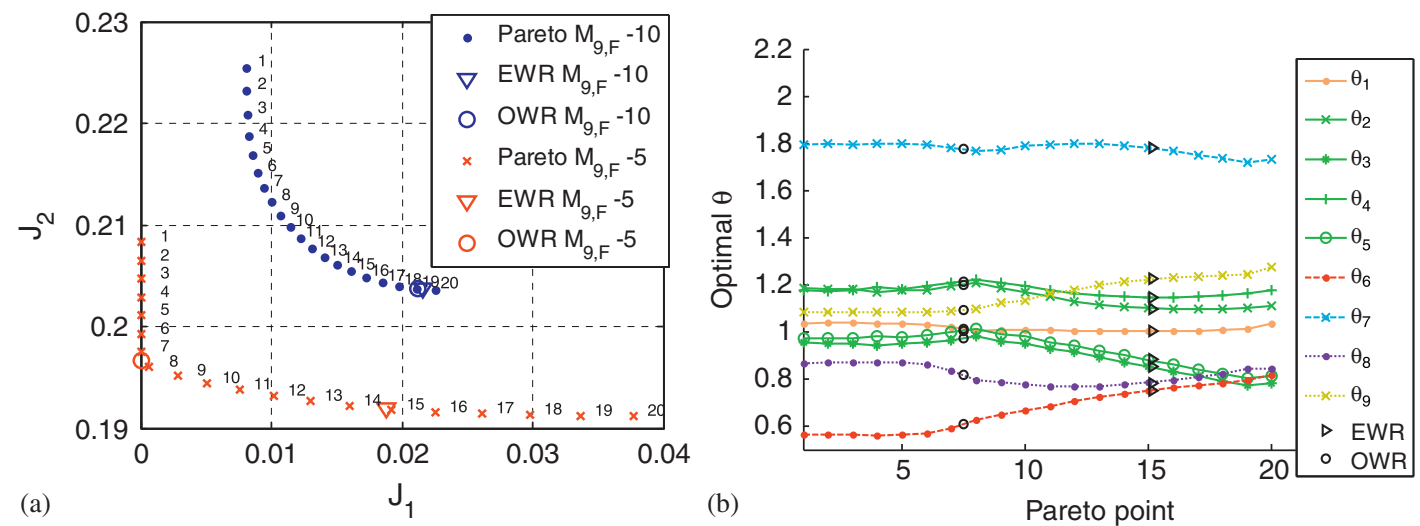

Figure 14. (a) Comparison of Pareto front for 5 and 10 modes for the nine-parameter fine model class $\mathrm{M}_{9, \mathrm{~F}}$ and (b) Pareto optimal solutions for the nine-parameter fine-mesh model class $\mathrm{M}_{9, \mathrm{~F}}$ for five modes.

part of the Pareto front that arises by minimizing the mode shape residuals $J_{2}(\underline{\theta})$. In contrast to the zero residuals predicted for the unidentifiable part from 1 to 7 , the residuals corresponding to the unidentifiable part from 17 to 20 are non-zero, indicating that there is a discrepancy between the measured mode shapes and the mode shapes predicted by the optimal set of unidentifiable models.

It is worth pointing out that the NBI algorithm can compute the unidentifiable solutions that occur by minimizing either the modal frequency or the mode shape residuals. Special algorithms have been proposed in the literature [17-18] to estimate such unidentifiable solutions. The NBI technique offers a computationally efficient alternative to compute such solutions since they are included in the NBI set of optimal solutions.

The solutions provided by the OWR and the EWR methods for the case of $M_{9, F^{-}}-5$ differ considerably. The solution provided from the OWR is very close to the left end of the Pareto front. This solution is nearly the same as the solution that will be obtained by minimizing only the objective $J_{1}(\underline{\theta})$ in the modal frequencies, ignoring the fit in the mode shape components. In contrast, the EWR is very close to the right end of the Pareto front. This solution is the same as the solution that one will obtain by minimizing only the objective $J_{2}(\underline{\theta})$ involving the mode shape components, ignoring the fit in the modal frequencies. Thus, in this case the OWR solution, which is the most probable solution from a Bayesian inference point of view [23], differs significantly from the EWR solution.

The corresponding variability of the Pareto optimal model parameters for the nine-parameter fine-mesh model class $M_{9, F}$ used to fit the five modes is shown in Figure 14(b). Comparing with Figure 11(c) for the variability of the nine-parameter fine-mesh model class $M_{9, F}$ used to fit the 10 modes, it is observed that the variability in the values of the parameters along the useful identifiable part of the Pareto front (solutions 7 to 16) is similar to that observed in Figure 11(c). For a number of model parameters, this variability for the case of 5 modes is slightly smaller than the variability for the case of 10 modes due to the smaller set of modal data that have to be traded-off in the updating.

\subsection{Effect of the number of measurements on Pareto front and optimal FE models}

The Pareto fronts obtained using 72 and 36 sensors are next compared in Figure 15(a) for the nine-parameter model class $\mathrm{M}_{9, \mathrm{~F}}$ updated using the ten lowest modes. The reduced number of 36 sensors is a subset of sensors involved in the 72 sensor configuration shown in Figure 3. The following observations are made. The Pareto solution 1 at the left end of the Pareto front corresponds to optimizing the objective in the modal frequencies, which is the same for both sensor configurations. Thus, as observed in Figure 15(a), these two solutions give exactly the same fit to the modal frequency residuals $J_{1}(\underline{\theta})$. The size of the identifiable parts of the Pareto fronts, ignoring the unidentifiable part from Pareto points 17 to 20 for the 36-sensor 

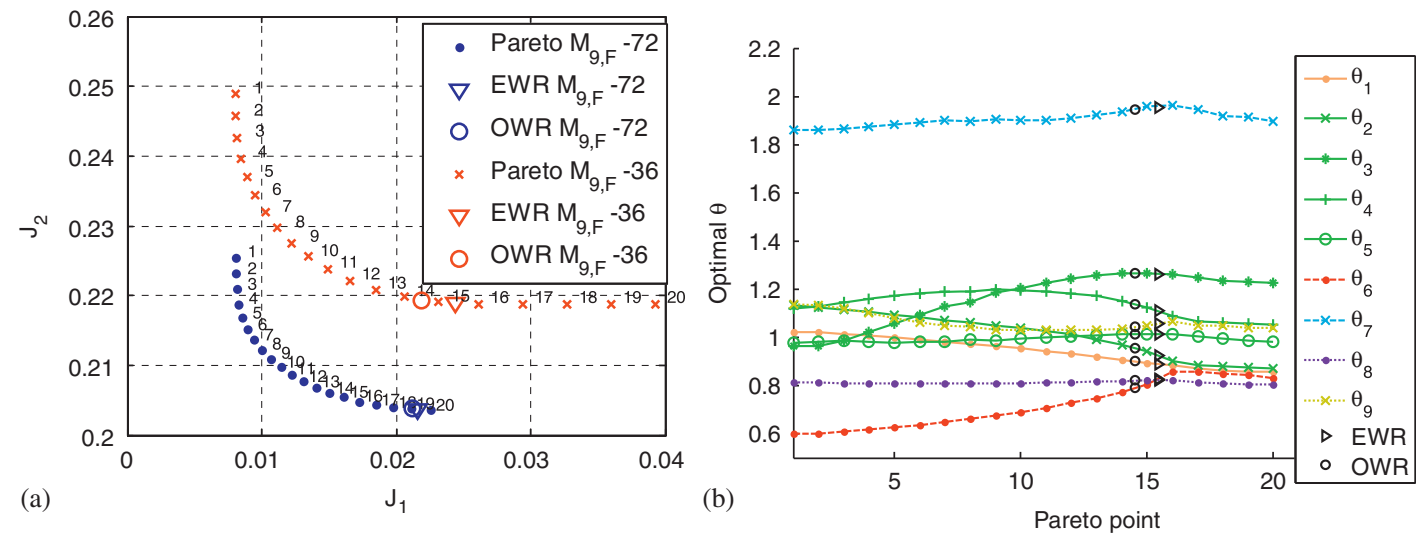

Figure 15. (a) Comparison of Pareto fronts for 72 and 36 sensors for the nine-parameter model class $\mathrm{M}_{9, \mathrm{~F}}$ and (b) Pareto optimal solutions for the nine-parameter model class $\mathbf{M}_{9, \mathrm{~F}}$ computed for 36 sensors.

configuration case, have approximately the same length for both cases. For all Pareto points, the solutions for the 72-sensor configuration seem to provide a better fit to the mode shape residuals $J_{2}(\theta)$. However, this is contrary to intuition since increasing the number of measurements from 36 to 72, a higher error should be expected for the same number of model parameters. This observed reduction in the $J_{2}(\underline{\theta})$ value is due to the normalization $\left\|\hat{\phi}_{r}\right\|$ of the mode shape error defined by (1), which is different for the two sensors configuration cases since it depends on the number of sensors used. Thus, the $J_{2}(\underline{\theta})$ objectives for the two sensor configurations differ and so a direct comparison of $J_{2}(\underline{\theta})$ values between sensor configurations involving different number of sensors is not meaningful.

The variability of the Pareto optimal parameter values for the 36-sensor configuration is shown in Figure 15(b) and should be compared to the corresponding variability of the 72-sensor configuration shown in Figure 11(c). Comparing the results of the identifiable part of the Pareto front (Pareto points 1 to 16) in Figure 15(b) with the results in Figure 11(c), it can be seen that the variability of the Pareto optimal solutions does not significantly depend on the number of sensors used in this work. The effect of the number of sensors is expected to be more significant as the number of sensors will decrease considerably.

\section{RESPONSE PREDICTION VARIABILITY FROM PARETO OPTIMAL FE MODELS}

The purpose of the model updating is to construct faithful structural models, within a selected model class, that can be used for making improved structural response predictions consistent with the measured data. The alternative Pareto optimal models obtained along the Pareto front provide different response predictions that are all acceptable based on the measured data and the measures of fit employed. The variability of the structural response predictions is next explored using two structural response performance indices: the FRF and the damage due to fatigue.

\subsection{Variability of FRF predictions}

The FRF predicted by the Pareto optimal solutions for the nine and three-parameter model classes $M_{9, F}$ and $M_{3, F}$ are compared in Figure 16(a, b) to the FRF computed directly from the measured data at sensor location 72 (see Figure 3 ) in the frequency range [20,90 Hz]. The FRF of the initial nominal model is also shown in Figure 16(a,b) to be inadequate to represent the measured FRF. Compared to the FRF of the initial nominal model, it is observed that the updated Pareto optimal models from the nine-parameter model class $M_{9, F}$ in Figure 16(a) tend to considerably improve the fit between the model predicted and the experimentally obtained FRF close to the resonance peaks. Also, it can be clearly seen that there is variability in the FRF predicted from the different Pareto optimal models 1, 10 and 20, which is due to the 

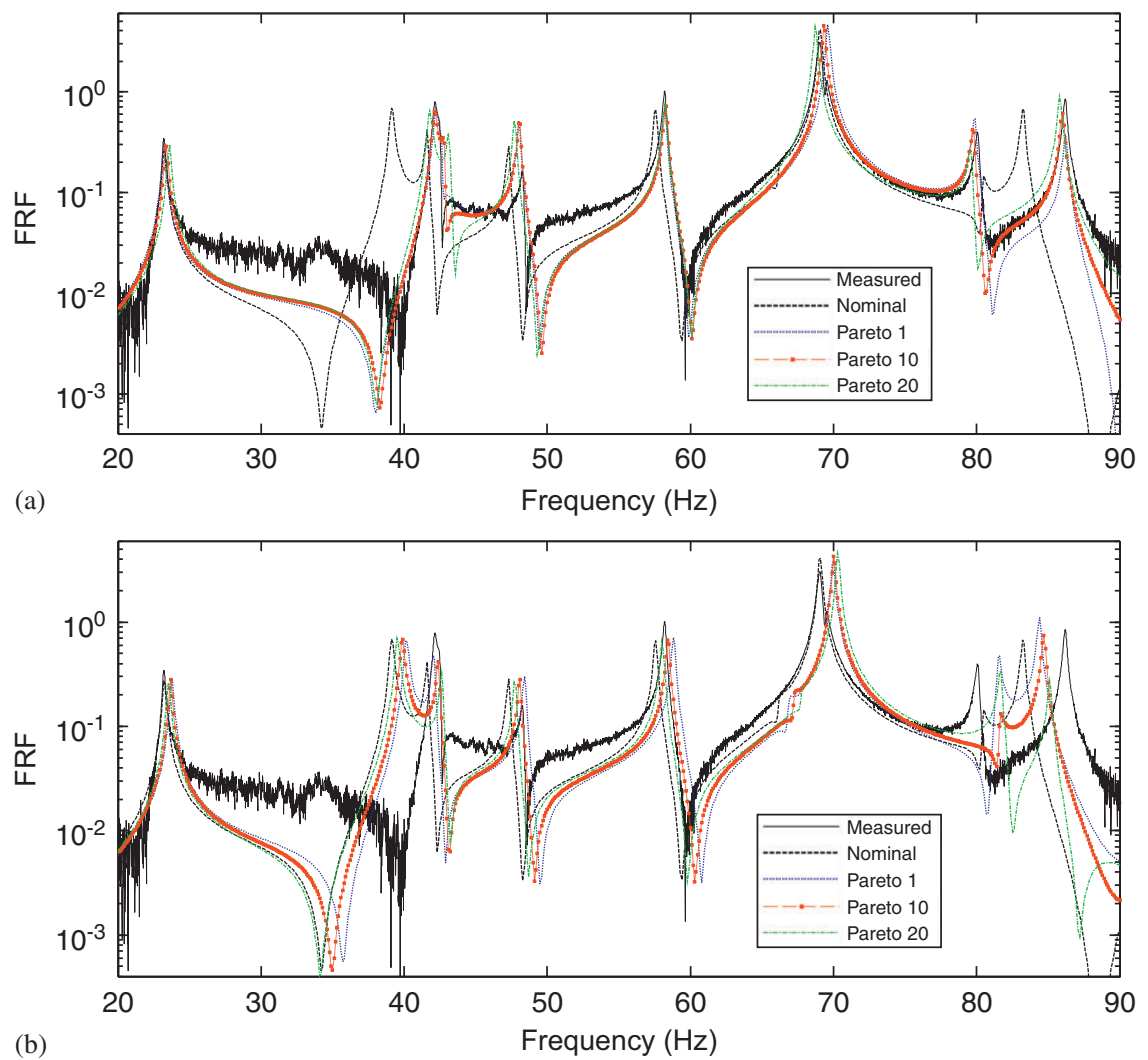

Figure 16. Comparison between measured, nominal and predicted FRF from the Pareto models 1,10 and 20 for the fine-mesh model class: (a) nine-parameter $\mathbf{M}_{9, \mathrm{~F}}$ and (b) three-parameter $\mathbf{M}_{3, \mathrm{~F}}$.

variability in the identified Pareto optimal models. This variability in FRF defines the uncertainty bounds in FRF predicted by the data-consistent Pareto optimal FE models.

Comparing Figure 16(a, b), a significant improvement in the FRF fit is clearly observed for the nine-parameter model class. The FRF predicted by the Pareto optimal models for the threeparameter model class $M_{3, F}$ in Figure $16(\mathrm{~b})$ tend to be closer to the FRF predicted by the nominal model than to the measured FRF. Thus, the Pareto optimal models from the threeparameter model class fail, in relation to the Pareto optimal models of the nine-parameter model class $M_{9, F}$, to give an adequate fit to the measured FRF, missing several of the resonance peaks.

\subsection{Variability of fatigue lifetime predictions}

Similar variability can be constructed for other structural performance indices. One important safety-related performance index for a metallic structure is the damage accumulation due to fatigue. The variability in the fatigue lifetime predictions at selected locations in the vehicle frame is next considered for stochastic excitation cases modeled by stationary Gaussian processes. These excitations for vehicles may arise from the spatial variability in road profiles. Available frequency domain methods based on spectral moments $[30,31]$ are used to predict the expected damage accumulation due to fatigue using the linear Palmgren-Miner rule $[32,33]$ and $S$ - $N$ fatigue curves obtained from laboratory experiments on simple specimens that are subjected to constant amplitude loads. The $S$ - $N$ curves describe the number of cycles $N(\sigma)=c \sigma^{-\alpha}$ required for failure in terms of the constant amplitude stress level $\sigma$. The parameters $c$ and $\alpha$ are constants obtained from fatigue test experiments and depend on the material and the type of the test specimen.

The frequency domain formulations for fatigue predictions depend on the probability distribution of stress cycles corresponding to different stress levels in a stress response time 
history. Various expressions for the probability distribution of stress cycles have been proposed in terms of the spectral moments of the stress process, which are readily obtained from the power spectral density of the stress components involved. This results in expected fatigue lifetime expressions in terms of the spectral moments of the stress process. Specifically, for uniaxial state of stress, the expected fatigue lifetime is given by

$$
T_{\text {life }}=\left[\bar{D}\left(\lambda_{0}, \lambda_{1}, \lambda_{2}, \lambda_{4}\right)\right]^{-1}
$$

where $\bar{D}\left(\lambda_{0}, \lambda_{1}, \lambda_{2}, \lambda_{4}\right)$ is the expected damage rate, $\lambda_{j}=\int_{-\infty}^{\infty}|\omega|^{j} S_{\sigma}(\omega) \mathrm{d} \omega, i=0,1,2,4$, are the spectral moments of the uni-axial stress $\sigma(t)$ that depend on the power spectral density (PSD) $S_{\sigma}(\omega)$ of the uni-axial stress $\sigma(t)$. For the important case of wide band processes, encountered often in applications, the widely used Dirlik approximation [34] is considered herein for modeling the probability of stress cycles in terms of the spectral moments of the stress process. In this case, the specific form of the expected damage $\bar{D}\left(\lambda_{0}, \lambda_{1}, \lambda_{2}, \lambda_{4}\right)$ in (10) with respect to the spectral moments can be found in [31].

For bi-axial state of stress, which is the case for the shell elements used in the present FE formulation, the fatigue lifetime prediction is computed using the equivalent stress concept introduced in [35]. Specifically, the fatigue lifetime is computed by (10) with the PSD $S_{\sigma}(\omega)$ of the uni-axial stress $\sigma(t)$ replaced by an equivalent PSD of the bi-axial stress tensor, given by

$$
S_{\text {equiv }}(\omega)=S_{\sigma_{x}}(\omega)+S_{\sigma_{y}}(\omega)-S_{\sigma_{x} \sigma_{y}}(\omega)+3 S_{\sigma_{x y}}(\omega)
$$

where $S_{\sigma_{x}}(\omega), S_{\sigma_{y}}(\omega)$ and $S_{\sigma_{x y}}(\omega)$ denote the power spectral density of the bi-axial stress tensor components $\sigma_{x}(t), \sigma_{y}(t)$ and $\sigma_{x y}(t)$, respectively, while $S_{\sigma_{x y}}(\omega)$ denotes the cross power spectral density matrix between the stress components $\sigma_{x}(t)$ and $\sigma_{y}(t)$.

The aforementioned methodology assumes that the power spectral density of the Gaussian stress process at a structural location is available. For linear structural behavior and Gaussian excitations, the components of the stress tensor at a structural location are also Gaussian processes with spectral moments that can be readily obtained by combining available random vibration techniques [36] with the FE model used to simulate the behavior of the structure.

For demonstration purposes it is assumed that the excitations at the four bases of the vehicle are fully correlated and modeled by a stationary white noise process. For the steel vehicle frame, the values of the fatigue constants are taken to be $c=4.06 \times 10^{88}$ and $a=9.82$. The variability of the fatigue lifetime predictions is computed at three different locations in the structure shown in Figure 3. For each location, the predictions of the variability are estimated for the model classes with three, six and nine parameters. The stress values at these three points are relatively high with respect to other points in the structure. All results assume modal damping values for all modes equal to $\zeta=0.02$. For comparison purposes, the number of contribution modes in the fatigue lifetime predictions is kept constant and equal to 10 .

Figure 17(a) compares at the three selected stress points the variability of the fatigue lifetime predictions obtained for the Pareto optimal FE models of the fine-mesh model classes $M_{3}, M_{6}$ and $\mathrm{M}_{9}$ identified using 10 modes and 72 sensors. Figure 17(b-d) shows results for the variability of the fatigue lifetime predictions for stress points 1, 2 and 3, respectively. Results in Figure 17(b-d) also include the fatigue lifetime prediction of the Pareto optimal models obtained from the model class $M_{9}$ identified using 5 modes and 72 sensors $\left(\right.$ Case $\left.M_{9}-5\right)$ and also from the model class $M_{9}$ identified using 10 modes and 36 sensors (Case $\left.M_{9}-36\right)$.

Comparing the variability of the Pareto optimal predictions at the different stress points, it can be said that the size of the variability depends on the location of the stress point, the parameterized model class used for predictions as well as on the data used to identify the model class. In general, the variability of the lifetime fatigue for stress points 1 and 2 is significantly higher than the variability for the stress point 3. Specifically, for the highest fidelity model class $\mathrm{M}_{9}$, the variability in the fatigue lifetime predictions is the highest at stress points 1 and 2, while it is significantly smaller at stress point 3 . The predictions of the Pareto optimal models from the model class $M_{3}$ as compared to the predictions of all other model classes underestimate the 

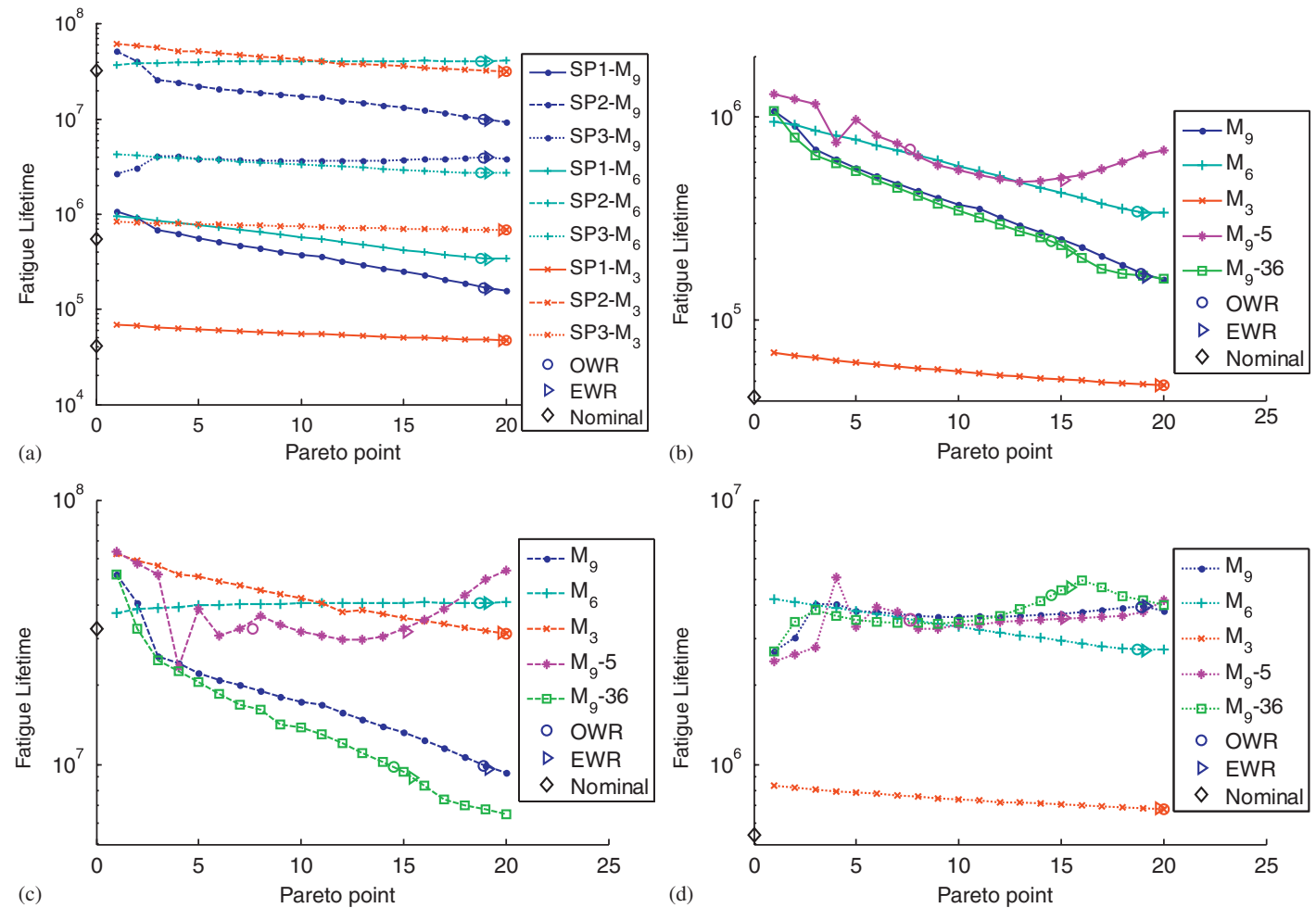

Figure 17. Variability in fatigue lifetime predictions using the nominal and the Pareto models for various updated cases of the fine-mesh model classes $\mathrm{M}_{\mathrm{F}}$ : (a) All stress points (SP); (b) SP 1; (c) SP 2; and (d) SP 3.

fatigue lifetime values by almost one order of magnitude for the stress points 1 and 3. Moreover, the variability in the predictions from the $M_{3}$ model class is significantly reduced compared to the variability from the predictions of the highest fidelity model class $\mathrm{M}_{9}$. The predictions from the model class $M_{6}$ for the stress points 1 and 3 are closer to the ones obtained from model class $M_{9}$, while the predictions for the stress point 2 do not vary significantly along the Pareto optimal models. The case $M_{9}-36$ gives almost the same variability in the fatigue lifetime predictions as the model class $\mathrm{M}_{9}$. The case $\mathrm{M}_{9}-5$ gives predictions that are comparable in values to those obtained from the model class $\mathrm{M}_{9}$ but the variability in these predictions is significantly smaller. Compared to the predictions of the Pareto optimal models, the predictions from the initial nominal FE model underestimate by more than one order of magnitude the fatigue lifetime at stress points 1 and 3 .

The predictions from the optimal models obtained from the OWR and EWR methods depend on the location of these points along the Pareto front. For all model identification cases involving 10 modes, these optimal models almost coincide along the Pareto front and therefore their predictions are shown to be very close in Figure 17. For the model identification case including 5 modes, the optimal models obtained by the OWR and the EWR methods differ significantly (Figure 14(a)) and thus their fatigue lifetime predictions are expected to differ as it is observed in Figure 17(b) for the case $\mathrm{M}_{9}-5$.

Concluding, the fatigue lifetime predictions from the Pareto optimal models may vary considerably, even by an order of magnitude. This implies that the widely used single (updated) FE model based on user selected weight values in WR methods is misleading and inadequate to represent the uncertain variability in the optimal FE models and their corresponding response predictions. The entire set of Pareto models provides this uncertainty in the optimal FE models as well as the uncertainty bounds in the predictions from these Pareto optimal models, consistent with the measured data and the norms used to measure the discrepancies between the measured modal values and the modal values predicted by the FE models. 


\section{CONCLUSIONS}

A multi-objective identification method was used to derive the entire set of the Pareto optimal FE models of a small-scale laboratory vehicle frame using its experimentally identified modal characteristics. In contrast to conventional WR methods, on which a single optimal FE model is promoted based on subjective choice of the weight values, the Pareto models contain all alternative FE models that are also derivable by the conventional WR method by varying the values of the weights from zero to infinity. The set of Pareto models quantifies the FE model uncertainty by providing all possible values of the model parameters given the measured data and the norms used to measure the discrepancies between the measured and model predicted modal characteristics. The NBI algorithm was demonstrated to be superior to the WR-based algorithms for solving the multi-objective optimization problem and effectively computing the useful identifiable part of the Pareto front and the Pareto optimal solution, as well as portions of the unidentifiable part of the Pareto front manifested at the edge points. The WR has certain disadvantages in adequately representing the Pareto front since for the same computational effort it may either miss significant portion of the Pareto front or promote non-interesting unidentifiable solutions encountered at the Pareto end points.

A wide variety of Pareto optimal structural models was obtained for the vehicle frame that trade-off the fit in the measured modal frequencies and mode shapes. This variability in the Pareto optimal solutions is due to model and measurement errors. The updated uncertain set of FE models was demonstrated to concentrate along certain manifolds in the parameter space. Using model-based simulations, the uncertainty in FE models can be propagated to predict the corresponding uncertainty in the response predictions simulated from the Pareto models. A parametric study on the vehicle structure has demonstrated that the variability in the Pareto optimal models depend, among other factors, on the size of the FEs selected to model the structure, the parameterization scheme used to define the number and type of parameters to be updated and the information contained in the measured data (number of sensors and the number of measured modes). Reducing the size of the FEs and increasing the number of model parameters results in higher fidelity models that tend to reduce the variability in the Pareto optimal models and improve the fit between FE model predicted and measured modal characteristics. The observed variability in the Pareto optimal vehicle models affects considerably the variability in the response predictions. FRFs and fatigue lifetime predictions have demonstrated that such variability may be significant and thus it should be taken into consideration in the decisions made based on the updated FE models. The use of a single model promoted by the conventional WR method is misleading and does not properly account for the uncertainty quantified by the entire set of data-consistent Pareto optimal solutions. The proposed multi-objective identification method provides the general framework for properly accounting for model uncertainty in response predictions based on measured data.

\section{ACKNOWLEDGEMENTS}

This research is part of the 03-ED-524 project, implemented within the framework of the 'Reinforcement Programme of Human Research Manpower' (PENED) and co-financed 25\% from the Greek Ministry of Development (General Secretariat of Research and Technology) and 75\% from E.U. (European Social Fund).

\section{REFERENCES}

1. Mottershead JE, Friswell MI. Model updating in structural dynamics: a survey. Journal of Sound and Vibration $1993 ; \mathbf{1 6 7 : 3 4 7 - 3 7 5 . ~}$

2. Papadimitriou C, Beck JL, Katafygiotis LS. Updating robust reliability using structural test data. Probabilistic Engineering Mechanics 2001;16:103-113.

3. Fritzen CP, Jennewein D, Kiefer T. Damage detection based on model updating methods. Mechanical Systems and Signal Processing 1998; 12(1):163-186. 
4. Teughels A, De Roeck G. Damage detection and parameter identification by finite element model updating. Archives of Computational Methods in Engineering 2005; 12(2):123-164.

5. Vanik MW, Beck JL, Au SK. Bayesian probabilistic approach to structural health monitoring. Journal of Engineering Mechanics (ASCE) 2000; 126:738-745.

6. Ntotsios E, Papadimitriou C, Panetsos P, Karaiskos G, Perros K, Perdikaris Ph. Bridge health monitoring system based on vibration measurements. Bulletin of Earthquake Engineering 2009; 7:469-483.

7. Metallidis P, Verros G, Natsiavas S, Papadimitriou C. Fault detection and optimal sensor location in vehicle suspensions. Journal of Vibration and Control 2003; 9:337-359.

8. Casciati S. Stiffness identification and damage localization via differential evolution algorithms. Structural Control and Health Monitoring 2008; 15:436-449.

9. Rahai A, Bakhtiari-Nejad F, Esfandiari A. Damage assessment of structure using incomplete measured mode shapes. Structural Control and Health Monitoring 2007; 14:808-829.

10. Yuen KV, Beck JL. Reliability-based robust control for uncertain dynamical systems using feedback of incomplete noisy response measurements. Earthquake Engineering and Structural Dynamics 2003; 32(5):751-770.

11. Haralampidis Y, Papadimitriou C, Pavlidou M. Multi-objective framework for structural model identification. Earthquake Engineering and Structural Dynamics 2005; 34(6):665-685.

12. Christodoulou K, Ntotsios E, Papadimitriou C, Panetsos P. Structural model updating and prediction variability using Pareto optimal models. Computer Methods in Applied Mechanics and Engineering 2008; 198(1):138-149.

13. Das I, Dennis Jr JE. Normal-boundary intersection: a new method for generating the Pareto surface in nonlinear multi-criteria optimization problems. SIAM Journal of Optimization 1998; 8:631-657.

14. Ntotsios E, Papadimitriou C. Multi-objective optimization algorithms for finite element model updating. International Conference on Noise and Vibration Engineering (ISMA2008), Katholieke Universiteit Leuven, Leuven, Belgium, 15-17 September 2008.

15. Nelson RB. Simplified calculation of eigenvector derivatives. AIAA Journal 1976; 14(9):1201-1205.

16. Beck JL, Katafygiotis LS. Updating models and their uncertainties-I: Bayesian statistical framework. Journal of Engineering Mechanics (ASCE) 1998; 124(4):455-461.

17. Katafygiotis LS, Papadimitriou C, Lam HF. A probabilistic approach to structural model updating. International Journal of Soil Dynamics and Earthquake Engineering 1998; 17:495-507.

18. Katafygiotis LS, Lam HF. Tangential-projection algorithm for manifold representation in unidentifiable model updating models. Earthquake Engineering and Structural Dynamics 2002; 31(4):791-812.

19. Moaveni B, He X, Conte JP, Restrepo JI. Damage identification study of a seven-story full-scale building slice tested on the UCSD-NEES shake table. Structural Safety 2010; 32(5):347-356.

20. Moaveni B, He X, Conte JP, De Callafon RA. Damage identification of a composite beam using finite element model updating. Computer-Aided Civil and Infrastructure Engineering 2008; 23(5):339-359.

21. Weber B, Paultre P, Proulx J. Structural damage detection using nonlinear parameter identification with Tikhonov regularization. Structural Control and Health Monitoring 2007; 14:406-427.

22. Yuen K-V, Beck JL, Katafygiotis LS. Efficient model updating and health monitoring methodology using incomplete modal data without mode matching. Structural Control and Health Monitoring 2006; 13:91-107.

23. Christodoulou K, Papadimitriou C. Structural identification based on optimally weighted modal residuals. Mechanical Systems and Signal Processing 2007; 21:4-23.

24. Teughels A, De Roeck G, Suykens JAK. Global optimization by coupled local minimizers and its application to FE model updating. Computers and Structures 2003; 81(24-25):2337-2351.

25. Katafygiotis LS. Treatment of model uncertainties in structural dynamics, Technical Report EERL91-01, California Institute of Technology, Pasadena, California, 1991.

26. Katafygiotis LS, Beck JL. Updating models and their uncertainties. II: model identifiability. Journal of Engineering Mechanics (ASCE) 1998; 124(4):463-467.

27. Giagopoulos D, Natsiavas S. Hybrid (numerical-experimental) modeling of complex structures with linear and nonlinear components. Nonlinear Dynamics 2007; 47:193-217.

28. Peeters B, Van der Auweraer H, Guillaume P, Leuridan J. The PolyMAX frequency-domain method: a new standard for modal parameter estimation? Shock and Vibration 2004; 11(2-4):395-409.

29. Comsol AB. 2005. COMSOL multiphysics user's guide. Available from: http://www.comsol.com/.

30. Lutes LD, Larsen CE. Improved spectral method for variable amplitude fatigue prediction. Journal of Structural Engineering (ASCE) 1990; 116(4):1149-1164

31. Benasciutti D, Tovo R. Spectral methods for lifetime prediction under wide-band stationary random processes. International Journal of Fatigue 2005; 27:867-877.

32. Palmgren A. Die Lebensdauer von Kugallagern. VDI-Zeitschrift 1924; 68(14):339-341.

33. Miner MA. Cumulative damage in fatigue. Applied Mechanics Transactions (ASME) 1945; 12(3):A159-A164.

34. Dirlik T. Applications of computers to fatigue analysis. Ph.D. Thesis, Warwick University, 1985.

35. Pitoiset X, Preumont A. Spectral methods for multiaxial random fatigue analysis of metallic structures. International Journal of Fatigue 2000; 22:541-550.

36. Lutes LD, Sarkani S. Random Vibrations: Analysis of Structural and Mechanical Systems. Elsevier ButterworthHeinemann: Oxford, 2004. 